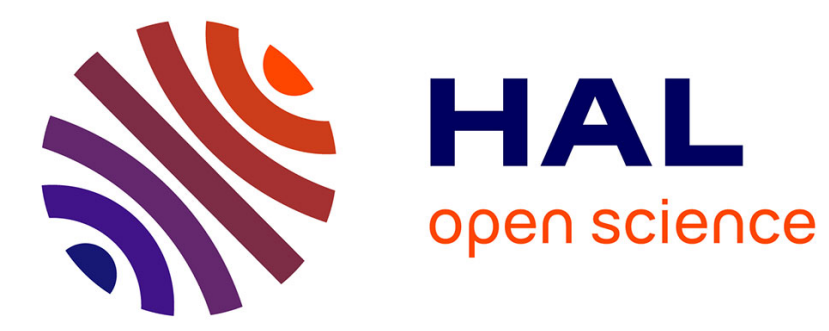

\title{
On the attainable set for a scalar nonconvex conservation law
}

Boris Andreianov, Carlotta Donadello, Andrea Marson

\section{To cite this version:}

Boris Andreianov, Carlotta Donadello, Andrea Marson. On the attainable set for a scalar nonconvex conservation law. 2016. hal-01346993

\section{HAL Id: hal-01346993 \\ https://hal.science/hal-01346993}

Preprint submitted on 20 Jul 2016

HAL is a multi-disciplinary open access archive for the deposit and dissemination of scientific research documents, whether they are published or not. The documents may come from teaching and research institutions in France or abroad, or from public or private research centers.
L'archive ouverte pluridisciplinaire HAL, est destinée au dépôt et à la diffusion de documents scientifiques de niveau recherche, publiés ou non, émanant des établissements d'enseignement et de recherche français ou étrangers, des laboratoires publics ou privés. 


\title{
On the attainable set for a scalar nonconvex conservation law
}

\author{
Boris P. Andreianov * Carlotta Donadello $^{\dagger}$ Andrea Marson $^{\ddagger}$
}

\begin{abstract}
We consider a Cauchy problem for a scalar conservation law in one space dimension with a compactly supported initial datum,

$$
\left\{\begin{array}{l}
\partial_{t} u+\partial_{x} f(u)=0 \\
u(0, x)=\left\{\begin{array}{lll}
u_{c}(x) & \text { if } & x \in[a, b], \\
0 & \text { if } & x \notin[a, b]
\end{array}\right.
\end{array}\right.
$$

Here $a, b \in \mathbb{R}$ are given, and the flux function $f$ is assumed to be non convex, in particular to have a single inflection point. We regard the function $u_{c}$ as a control. The main results of the paper states sufficient conditions for a function $v$ to be attained at time $T$ by a trajectory of $(*)$.
\end{abstract}

Mathematical Subject Classification: 35Q93, 35L65

Key Words: scalar conservation laws, nonconvex flux, control

\section{Introduction}

Aim of this paper is to initiate the description of the set $\mathfrak{A}_{T}$ of profiles $v$ in $B V(\mathbb{R})$ which can be attained at a fixed time $t=T$ by the entropy admissible solution of

$$
\begin{aligned}
& \partial_{t} u+\partial_{x} f(u)=0, \\
& u(0, x)=\left\{\begin{array}{lll}
u_{c}(x) & \text { if } & x \in[a, b] \\
0 & \text { if } & x \notin[a, b]
\end{array}\right.
\end{aligned}
$$

where $a<b$ are given, and $u_{c} \in L^{\infty}(a, b)$ is regarded as a control. We assume the flux function $f$ to fulfill the set of conditions

\footnotetext{
*Laboratoire de Mathématique et Physique Théorique, Université de Tours, Parc de Grandmont, 37200, Tours, France; e-mail: boris.andreianov@lmpt.univ-tours.fr

${ }^{\dagger}$ Laboratoire de Mathématique de Besançon, Université de Franche-Comté, 16 Route de Gray, 25030, Besançon, France; e-mail: carlotta.donadello@univ-fcomte.fr

${ }^{\ddagger}$ Dipartimento di Matematica, Via Trieste 63, 35121 - Padova, Italy, and Laboratoire de Mathématique de Besançon, Université de Franche-Comté, 16 Route de Gray, 25030, Besançon, France; e-mail: marson@math.unipd.it
} 
(F) a. $f$ is of class $C^{2}$,

b. $f(0)=f^{\prime}(0)=f^{\prime \prime}(0)=0 \quad u f^{\prime \prime}(u)<0 \quad \forall u \neq 0$,

c. $f$ has superlinear growth at $\pm \infty$.

A family of classical examples is given by $f(u)=-|u|^{m} u, m \geq 2$. We stress that the conditions in $(\mathbf{F}) \mathbf{b}$. are not necessary to achieve the results in this paper but allow for a lighter presentation. In Section 4 we apply our results to the classical sedimentation model proposed by Kynch in [17] and we consider the flux function $f(u)=-u(1-u)^{2}$ which does not satisfy (F)b..

In the existing literature we can distinguish essentially three approaches toward the study of exact controllability and characterization of the set of attainable profiles for the equation (1.1a).

The pioneering paper by Ancona and the third author, [3], focuses on the exact controllability for the initial boundary value problem for (1.1a) in the case of strictly convex flux. The authors use the theory of generalized characteristics introduced by Dafermos in [10], and describe for any given time $T>0$, the set of states which are attainable in time $T$ starting from the initial condition $u_{0}=0$ by a control on the boundary data alone. Under similar hypothesis, Adimurthi, Ghoshal and Gowda [1,2] exploit the explicit representation of solutions given by the Lax-Oleinik formula to construct an explicit backward solver and give a coincise characterization of the set of attainable profiles for the initial value problem and the boundary value problem in the half-space and in a strip with two boundaries. Using again the method of generalized characteristics, Corghi and the third author characterize in [8] the attainable set for a scalar balance law with strictly convex flux

$$
u_{t}+f(u)_{x}=z(t, x), \quad \text { for } t \in[0, T], \quad \text { and } x \in \mathbb{R},
$$

where the right hand side $z$ acts as distributed control.

In the classical paper [15], Horsin obtains approximate controllability results for scalar conservation laws by a different approach, which relies on the return method introduced by Coron [9]. This technique is also an important ingredient in the result by Chapouly, [7], concerning the attainability of $C^{1}$ profiles for classical solutions of the balance law (1.2) on the strip $[0, T] \times[0,1]$. In this paper the boundary data at $x=0$ and $x=1$ act as controls together with the source term $z$ (distributed control) which only depends on the time variable t. A similar result, in the setting of entropy weak solution is due to Perrollaz, [19].

The return method has been applied in combination with the vanishing viscosity approach by Léautaud, [18], to study the uniform controllability of scalar conservation laws. This last paper is also the only one, to our knowledge, in which the flux function $f$ is allowed to have a finite number of inflection points. Starting from an initial condition $u_{0}$ in $L^{\infty}([0,1])$ and using the boundary data at $x=0$ and $x=1$ as controls, the author proves the attainability in time $T$ (depending on the $L^{\infty}$ norm of $u_{0}$ ) of constant states.

Finally, let us mention that for the viscous Burgers equation, constant states reachable by boundary controls has been studied using the Cole-Hopf transformation by Glass and Guerrero [12], Guerrero and Imanuvilov [13]. 
The results in the present paper are all obtained by an extensive application of the method of generalized characteristics. This approach naturally leads to a very fine analysis of the structure of solutions which allows us to give a rather complete insight of the possible obstructions to attainability. Moreover, we succeed in providing a complete explicit backward reconstruction of the solution for a significant class of attainable profiles, which contains all basic situations. However, even if it is certainly possible to use our results as building blocks toward the analysis of more complex cases, our work fails to provide an easy to check routine to distinguish attainable states from non attainable ones.

\subsection{Fine structure of solutions and obstacles to backward reconstruction}

We recall the definition of entropy admissible solution to (1.1a)-(1.1b) (see [11]).

Definition 1.1. An entropy admissible solution to (1.1a)-(1.1b) in the time interval $[0, T]$ is a continuous function $u:[0, T] \rightarrow L_{\text {loc }}^{1}(\mathbb{R})$ which assumes the initial datum $(1.1 \mathrm{~b})$, is a distributional solution to (1.1a) in $] 0, T[\times \mathbb{R}$, and satisfies an entropy admissibility condition (see [11]), i.e. for any entropy-entropy flux pair $(\eta, q)$, with $u \mapsto \eta(u)$ convex, there holds

$$
\left.\partial_{t} \eta(u)+\partial_{x} q(u) \leq 0, \quad \text { in }\right] 0, T[\times \mathbb{R},
$$

in the sense of distribution.

Throughout our paper, without loss of generality, we assume that the solutions are normalized to be left continuous.

In general, distributional solutions to (1.1a) are not smooth, regardless the possible regularity of the initial condition. The demise of classical solutions coincides with the appearance of jump discontinuities. The method of generalized characteristics has been introduced by Dafermos in [10] to investigate the fine structure of the solutions to (1.1a).

Definition 1.2. Let $u \in C\left([0, T] ; L_{l o c}^{1}(\mathbb{R})\right)$ be an entropy admissible solution of (1.1a).

- A classical characteristic associated to $u$ is a curve $\xi:\left[t_{0}, t_{1}\right] \rightarrow \mathbb{R}^{2}$ such that for some $\mathfrak{u}$ and for all $t \in\left[t_{0}, t_{1}\right]$ there holds

$$
\xi(t)=\xi\left(t_{0}\right)+f^{\prime}(\mathfrak{u})\left(t-t_{0}\right), \quad u(t, \xi(t))=\mathfrak{u} .
$$

- A generalized characteristic associated to $u$ is a Lipschitz continuous curve $\xi:\left[t_{0}, t_{1}\right] \rightarrow$ $\mathbb{R}^{2}$ which satisfies the differential inclusion

$$
\frac{d \xi}{d t}(t) \in I\left[f^{\prime}\left(u\left(t, \xi(t)^{+}\right)\right), f^{\prime}(u(t, \xi(t)))\right],
$$

where $I[a, b]$ denotes the closed interval of extrema $a$ and $b$.

Any classical characteristic is a generalized characteristic. 
Through any point of jump discontinuity of $u$ passes a funnel of backward generalized characteristic. The core of the results in [10, Section 2], concerns the study of the special properties of minimal and maximal backward generalized characteristics through a point. To limit the length of our manuscript, we decided not to recall all of these results but rather to give precise references at any time we apply them.

We recall that the method of generalized characteristics is fully developed only for the case in which the flux function $f$ admits at most one inflection point. A classical observation due to Hoff [14] is that, whenever the flux function $f$ admits more than one inflection point, the interaction between two discontinuity in the solution $u$ to (1.1a) can produce outgoing centered rarefaction waves at positive times, and this would make the analysis far more intricate

Once a jump discontinuity has appeared in the solution it persists, and its location describes a Lipschitz continuous curve $x=\varphi(t)$ in the $(t, x)$-plane. From the definition of distributional solution, one can check that the values of the left and right traces of the solution $u$ on the sides of $\varphi$ must satisfy the Rankine-Hugoniot conditions

$$
f(u(t, \varphi(t)+))-f(u(t, \varphi(t)))=\dot{\varphi}(t)[u(t, \varphi(t)+)-u(t, \varphi(t))] .
$$

Additionally, if the solution $u$ is entropy admissible, the following inequalities are satisfied (Lax-Oleinik conditions)

$$
f^{\prime}(u(t, \varphi(t))) \geq \dot{\varphi}(t) \geq f^{\prime}(u(t, \varphi(t)+)), \quad x=\varphi(t) .
$$

This imply that the curves of discontinuity are generalized characteristics.

If the flux function $f$ in (1.1a) if strictly convex, then both the inequality in (1.5) are strict, while a classical lemma due to Dafermos, [10, Lemma 2.2], specifies that under the assumptions $(\mathbf{F})$, only the second inequality above is strict. In this framework we can distinguish two kinds of discontinuities propagating in the solution:

- genuine shocks (which we call shocks in the following), for which both inequalities in (1.5) are strict;

- left contact discontinuities, for which there holds $\dot{\varphi}(t)=f^{\prime}(u(t, \varphi(t)))$.

As in the convex case, characteristics run into genuine shocks from both sides, while it may happen that characteristics radiate from one of the sides of a contact discontinuity.

The presence of contact discontinuities is responsible for the two major obstacles toward the formulation of a fast and easy to apply attainability test. First recall that in the convex case, whenever the target profile $v$ suffers from an admissible jump at $x=\bar{x}$ joining the states $v_{L}$ and $v_{R}$, there exist infinitely many ways to reconstruct backwardly a solution to (1.1a) in the triangular region delimited by the minimal and the maximal backward characteristics

$$
\eta_{\bar{x}}^{-}(t)=\bar{x}+f^{\prime}\left(v_{L}\right)(t-T), \quad \eta_{\bar{x}}^{+}(t)=\bar{x}+f^{\prime}\left(v_{R}\right)(t-T),
$$

respectively. This fact can be exploited to produce convenient backward reconstructions, see for example [4], and it is related to the dissipative properties of admissible shocks. 
On the contrary, since left contact discontinuities must satisfy the Rankine-Hugoniot condition together with $\dot{\varphi}(t)=f^{\prime}\left(v_{L}\right)$, it is clear that given the left state of a left contact discontinuity, the corresponding right state is immediately determined from the fact that the chord joining $\left(v_{L}, f\left(v_{L}\right)\right)$ to $\left(v_{R}, f\left(v_{R}\right)\right)$ needs to have slope exactly equal to $f^{\prime}\left(v_{L}\right)$. We call $\left(v_{L}\right)^{\sharp}$ the unique possible right state connected to $v_{L}$ by a left contact discontinuity. This means that there is no freedom in the backward reconstruction of the solution in an immediate right neighbourhood of a contact discontinuity.

We are now ready to illustrate the first obstacle in our work.

- Assume that the target profile $v$ suffers from a jump discontinuity at $x=\bar{x}$, joining the states $v_{L}$ and $v_{R}=\left(v_{L}\right)^{\sharp}$, and that the candidate backward characteristics from the points in a left neighbourhood of $\bar{x}$ cross one another at positive times. If $v$ is admissible, their tangent envelope must be a convex Lipschtz curve $t \mapsto \varphi(t)$ (see [10, Theorem 2.2]), and in this case our backward reconstruction contains a left contact discontinuity traveling along the curve $\varphi$. This geometric requirement translates into the fact that the attainability of $v$ may depend on the values of $v, f^{\prime}(v)$ and (as we show in Section 2) $f^{\prime \prime}(v)$ on a whole left neighbourhood of $\bar{x}$ and not just on the values of $v\left(\bar{x}^{-}\right)$and $v\left(\bar{x}^{+}\right)$. Of course, the presence of several jumps in the target profile may ask for additional compatibility conditions.

The second difficulty we face is the following.

- Once we detect the presence of a left contact discontinuity $\varphi$ in our backward reconstruction, we must trace back in a unique way the candidate backward characteristics from $(t, \varphi(t))$, that we denote by $\xi_{\varphi(t)}$. It might happen that the curves $\xi_{\varphi(t)}$ cross one another at positive times and have a convex tangent envelope $t \mapsto \varphi_{2}(t)$. In this case we continue our backward reconstruction including a second left contact discontinuity in the solution. Again, the values on the left and on the right of $\varphi_{2}$ are completely determined from the values of $v$ and we have no choice in our reconstruction. We know from the results in [10] that, if $v$ is an admissible target profile, this situation can occur a finite number of times before we reach $t=0$. We call this structure a nesting of contact discontinuities.

Even in the most simple case, which is briefly described in Section 5, the reconstruction of a solution in presence of a nesting structure is a delicate matter, which leads us to look for solutions of a characteristic boundary value problem in which the boundary datum is not to be taken in the usual hyperbolic sense [5], but should be imposed as the value of the trace of the solution along the boundary curve, see Section 5.2.

\subsection{Outline of the paper}

Notations are introduced in the last part of this Introduction. Section 4 contains an example related to a model of sedimentation. A direction of perspective research is outlined in Subsection 5.3. In the hope to palliate the technicality of the presentation, we give here a rather detailed summary of the results in Sections 2, 3 and 5 . 
Section 2: necessary conditions. Theorem 1 states necessary conditions toward attainability which are reminiscent of the classical necessary conditions for the convex flux case. Proposition 2.1 rules out the presence of centered rarefaction waves focusing at positive time. Proposition 2.2 is the first evidence of the strong rigid structure of solutions next to a left contact discontinuity. Basically, on the right of any left contact discontinuity curve $t \mapsto \varphi(t)$ we can define a "triangular" region delimited by $\varphi$, the maximal backward characteristic from the point of jump of $v, t \mapsto \eta_{\bar{x}}^{+}(t)$, and the line $t=0$. In this region the solution can be reconstructed in a unique way by following the backward characteristics steaming from the right side of the discontinuity. If nesting occurs, it must take place outside this region. Starting from this point we limit our attention to situations in which nesting does not occur, deferring to Section 5 some examples and comments concerning nesting.

Given a continuous target profile $v$ and a time $T$ we define (candidate) backward characteristics lines as in (1.14c). Proposition 2.3 gives a necessary condition for this family of lines to have a convex tangent envelope. This implies a geometric requirement for the attainability of $v$ because the characteristics associated to any solution to (1.1a) can cross only in a centered rarefaction wave at time $t=0$, or they can radiate from a left contact discontinuity, which is a convex curve.

Proposition 2.4 puts together the results in Proposition 2.2 and Proposition 2.3 to formulate a necessary condition for a contact discontinuity in $v$ to be admissible. Observe that while the admissibility of a shock discontinuity in the convex case is easily determined by the values of $v$ on the left and on the right sides of the jump, here we need to impose conditions on the first and second derivatives of $f^{\prime}(v)$ on a whole neighbourhood of the point of jump.

Section 3: sufficient conditions. The main result in this section is Theorem 2, which collects a set of sufficient conditions for the attainability of a profile $v$ in time $T$ provided that the points at which $v$ is discontinuous do not accumulate and that no nesting occurs. The first part of Section 3 consists of the statement of the theorem and of some remarks illustrating the meaning of the conditions. In the hypothesis of Theorem 2 it is possible to determine whether the profile $v$ is attainable by checking that each of its discontinuities is admissible. Therefore in Section 3.1 we detail the study of three basic situations which will be used as building blocks in the proof of Theorem 2. Lemmas 3.1, 3.2 and 3.3 explain how to construct a control which allows to reach the profile $v$ in three basic situations. The last part of Section 3 contains the proof of Theorem 2 .

Section 5: one example of nesting, a different point of view. Section 5.1 contains the analysis of the simplest possible case of nesting. Some conditions for attainability are states, in a very smooth framework. One explicit example illustrates our results. Section 5.2 is devoted to present a slightly different approach to the problem of nesting.

\subsection{Notation}

First of all, if a solution to (1.1a) suffers from jump discontinuity along a Lipschitz curve $x=\varphi(t)$, we denote by $u_{L}(t)$ and $u_{R}(t)$, respectively, the left and right limits of $u(t, \cdot)$ at $\varphi(t)$ 
whenever they exist, i.e.

$$
u_{L}(t)=\lim _{x \rightarrow \varphi(t)-} u(t, \cdot), \quad u_{R}(t)=\lim _{x \rightarrow \varphi(t)+} u(t, \cdot) .
$$

Let $f$ be a flux function satisfying the set of conditions $(\mathbf{F})$. Given $v \in \mathbb{R}, v \neq 0$, we let $v^{\sharp}$ be the solution to

$$
f\left(v^{\sharp}\right)=f(v)+f^{\prime}(v)\left(v^{\sharp}-v\right), \quad v^{\sharp} \neq v,
$$

so that

$$
f^{\prime}(v)=\frac{f(v)-f\left(v^{\sharp}\right)}{v-v^{\sharp}},
$$

holds for any $v \neq 0$. Symmetrically, we denote by $v^{b}$ the solution to

$$
f(v)=f\left(v^{b}\right)+f^{\prime}\left(v^{b}\right)\left(v-v^{b}\right), \quad v^{b} \neq v,
$$

so that

$$
f^{\prime}\left(v^{b}\right)=\frac{f(v)-f\left(v^{b}\right)}{v-v^{b}},
$$

holds for any $v \neq 0$. Observe that

$$
\begin{gathered}
v=\left(v^{\sharp}\right)^{b}=\left(v^{b}\right)^{\sharp}, \\
v \cdot v^{\sharp}<0, \quad v \cdot v^{b}<0,
\end{gathered}
$$

hold for any $v \neq 0$.

Throughout our paper we assume that the target profile $v$ is a left continuous BV function with compact support. We define

$$
\alpha \doteq \sup \{x \in \mathbb{R}: v(y)=0 \forall y \leq x\},
$$

so that $v(\alpha)=0$. Moreover, if $T$ is the fixed time at which $v$ should be attained, we let

$$
\begin{aligned}
& \eta_{x}^{-}(\tau) \doteq x+f^{\prime}(v(x))(\tau-T)=x+f^{\prime}(v(x-))(\tau-T), \\
& \eta_{x}^{+}(\tau) \doteq x+f^{\prime}(v(x+))(\tau-T),
\end{aligned}
$$

be respectively the (candidate) minimal and maximal backward characteristics lines from $(T, x)$ associated to a solution $u$ of (1.1a) attaining the profile $v$ at time $T$, i.e. $u(T, x)=v(x)$. In case $v$ is continuous at $x$, minimal and maximal backward characteristics coincide in a left neighbourhood $] T-\delta, T]$ of $t=T$, and therefore we can write

$$
\eta_{x}(\tau) \doteq x+f^{\prime}(v(x))(\tau-T),
$$

where $\tau \in] T-\delta, T]$.

The notations $D^{+}$and $D^{-}$stand for the right and left upper Dini derivatives respectively

$$
D^{+} g(x)=\limsup _{y \rightarrow x^{+}} \frac{g(y)-g(x)}{y-x}, \quad D^{-} g(x)=\limsup _{y \rightarrow x^{-}} \frac{g(y)-g(x)}{y-x} .
$$

Given any two points $a$ and $b$ in $\mathbb{R}$, we use the notation $I(a, b)$ to indicate the open interval of extrema $a$ and $b$. This means that $I(a, b)=] a, b[$ if $a<b$ and $I(a, b)=] b, a[$ if $a>b$. Analogously, $I[a, b]$ denotes the closed interval of extrema $a$ and $b$. 


\section{Necessary conditions for attainability}

Throughout this section we assume that $v$ is the value, at time $t=T$, of an entropy solution to (1.1a) defined on $[0, T]$. We analyze the necessary properties of $v$ due to this fact, using the backward characteristics as the main tool. The conditions in the following theorem are the exact counterpart of the necessary conditions for attainability stated in [3] for the convex flux case. They differ from all other conditions stated in this work because they depend on the value (or the limit value) of the target profile at single points.

Theorem 1. Assume that $v$ is a measurable, left continuous, bounded attainable profile, with finite total variation, so that there exists $u_{c} \in L^{\infty}(a, b)$ such that the weak entropy solution $u=u(t, x)$ to (1.1a)-(1.1b) fulfills $u(T, \cdot)=v$. Let $\alpha$ be defined as (1.13). Then the following properties hold.

1. Let $x_{0} \in \mathbb{R}$ be given and assume that $v\left(x_{0}+\right) \neq 0$. Then the candidate maximal backward characteristic from the point $\left(T, x_{0}\right), t \mapsto \eta_{x_{0}}^{+}(t)$, satisfies

$$
\eta_{x_{0}}^{+}(0)=x_{0}-f^{\prime}\left(v\left(x_{0}+\right)\right) T \leq b .
$$

2. Assume that $v(\alpha+) \neq 0$. Then the candidate maximal backward characteristic from the point $(T, \alpha), t \mapsto \eta_{\alpha}^{+}(t)$, satisfies

$$
\eta_{\alpha}^{+}(0)=\alpha-f^{\prime}(v(\alpha+)) T \geq a .
$$

3. Assume that $v(\alpha+)=0$. Then there exists a sequence $\left\{x_{n}\right\}_{n \in \mathbb{N}}$ such that $x_{n} \downarrow \alpha$ and for any $n \in \mathbb{N}$, the candidate maximal backward characteristic from the point $\left(T, x_{n}\right)$, $t \mapsto \eta_{x_{n}}^{+}(t)$, satisfies

$$
\eta_{x_{n}}^{+}(0)=x_{n}-f^{\prime}\left(x_{n}+\right) T>a .
$$

4. Assume that at $x=\bar{x}$ the function $v$ suffers from a jump discontinuity with left and right states $v_{L}=v(\bar{x})$ and $v_{R}=v(\bar{x}+)$, respectively. Then the following conditions on $v_{L}$ and $v_{R}$ hold.

(a) $f^{\prime}\left(v_{L}\right)>f^{\prime}\left(v_{R}\right)$;

(b) if $v_{L} \cdot v_{R}<0$, then let $v_{L}^{\sharp}$ be as in (1.7) with $v=v_{L}$. Then we have

$$
v_{R} \notin I\left(v_{L}, v_{L}^{\sharp}\right) .
$$

Proof. 1. Assume that (2.1) fails, and let $y=\zeta_{x_{0}}^{+}(t)$ be the maximal generalized backward characteristic from $\left(T, x_{0}\right)$ Since $y=\zeta_{x_{0}}^{+}(t)$ is a convex polygonal [10, Theorem 2.1], we have

$$
\zeta_{x_{0}}^{+}(t) \geq x_{0}+f^{\prime}\left(v\left(x_{0}+\right)\right)(t-T)=\eta_{x_{0}}^{+}(t),
$$

with $\eta_{x_{0}}^{+}(\cdot)$ be defined at (1.14c) (candidate backward characteristic). Since $u(0, x)=0$ for any $x>b$, in particular we have $u(0, x)=0$ for all $x \geq \zeta_{x_{0}}^{+}(0)$, so that $u(t, x)=0$ for any $x \geq \zeta_{x_{0}}^{+}(t)$ and any $t \leq T$. Hence $v\left(x_{0}+\right)=0$, contrary to the assumption. 
2. Assume by contradiction that (2.2) fails, and, to fix the ideas, assume $v(\alpha+)>0$. Let $x_{1}>\alpha$ be such that $v(x)>0$ for any $\alpha<x<x_{1}$. Then, there exists $\delta>0$ such that in the region

$$
\left\{(\tau, x): T-\delta \leq \tau \leq T, x \leq x_{1}+f^{\prime}\left(\left(v\left(x_{1}\right)\right)(\tau-T)\right\}\right.
$$

there holds $u(\tau, x) \geq 0$. Hence $u$ is a solution to a conservation law with convex flux. Since arguing as before we get $u(\tau, x)=0$ for any $x<\alpha+f^{\prime}(v(\alpha+))(\tau-T)$, it turns out that $u(T, \cdot)$ can not suffer from a jump discontinuity at $x=\alpha$.

3. Again, we argue by contradiction, and assume that there exist $\bar{x}>\alpha$ such that

$$
x-f^{\prime}(v(x+)) T \leq a \quad \forall \alpha<x \leq \bar{x} .
$$

Let $\eta_{x}(\cdot)$ be defined as at $(1.14 \mathrm{c})$. Two cases may occur.

(a) All of the lines $\eta_{x}(\cdot), \alpha<x \leq \bar{x}$, focus at $(0, a)$, i.e.

$$
x-f^{\prime}(v(x+)) T=a \quad \forall \alpha<x \leq \bar{x} .
$$

In this case the solution $u$ would contain a centered rarefaction wave with 0 left state, leading to a contradiction.

(b) If some of the lines $\eta_{x}(\cdot), \alpha<x \leq \bar{x}$, do not focus at $(0, a)$, we may assume that

$$
x-f^{\prime}(v(x+)) T<a \quad \forall \alpha<x \leq \bar{x}
$$

holds. But then, arguing as above, we can deduce that $v(y)=0$ in a right neighbourhood of $x=\alpha$, contrary to definition (1.13).

4. The proof of such properties of admissible jump discontinuities for a weak entropy solution to (1.1a) is classical (e.g., see [11, Chapter 8]).

As we limit our attention to the case in which the flux function $f$ in (1.1a) has exactly one inflection point we know that no rarefaction wave can be produced at positive time by the interaction of two or more wave-fronts. Therefore, any candidate target profile $v$ whose candidate backward characteristics focus at a single point of $\mathbb{R}^{+} \times \mathbb{R}$ is not attainable. This fact motivate the following Proposition.

Proposition 2.1. Assume that $v$ is an attainable profile, continuous in an interval $I$, with $D_{x} f^{\prime}(v(x))=c>0^{1}$ for any $x \in I$. Then $c \leq 1 / T$.

Proof. If $D_{x} f^{\prime}(v(x))=c$ for all $x \in I$ then the lines $\eta_{x}$, defined as in (1.14c) for $x \in I$, take the form

$$
\eta_{x}(\tau)=x+c(x+a)(\tau-T),
$$

where $a$ is a constant, and intersect at $\tau=T-1 / c, y=-a$. Since in any solution to (1.1a) no new rarefaction are created at positive times, $\tau \leq 0$ must hold.

\footnotetext{
${ }^{1}$ Here $D_{x} f^{\prime}(v(x))$ denotes the derivative of the function $x \mapsto f^{\prime}(v(x))$.
} 
Now let $u$ be a bounded weak entropy solution to (1.1a) suffering from a discontinuity along a time-like curve $x=\varphi(t)$, for $t \in\left[t_{0}, t_{1}\right]$, with right state state $u_{R}(t)$ (see (1.6)). The (candidate) maximal backward characteristic curve from the point $(t, \varphi(t))$ is given by the equation

$$
\xi_{t}(\tau)=\varphi(t)+f^{\prime}\left(u_{R}(t)\right)(\tau-t)
$$

We stress that all through the paper, for the reader convenience, backward characteristics originated at time $T$ are denoted by the greek letter $\eta$, while backward characteristics originated at earlier times, steaming from a curve of discontinuity in the solution $u$, are denoted by the greek letter $\xi$. It is possible, of course, that some of the candidate backward characteristics $\xi_{t}, t \in\left[t_{0}, t_{1}\right]$, cross each other. This is at the origin of the nesting phenomenon evoked in the Introduction. However, the next Proposition shows that the lines $\xi_{t}$ do not intersect in the region between $\varphi$ and $\xi_{t_{1}}$.

Proposition 2.2. Assume that $u$ is a bounded measurable weak entropy solution to (1.1a) suffering from a left contact discontinuity $x=\varphi(t), t \in\left[t_{0}, t_{1}\right]$, with right state $u_{R}(t)$. Let $\xi_{t}(\cdot)$ be as at $(2.5)$, and assume that $\xi_{t}(\tau) \leq \xi_{t_{1}}(\tau)$ for any $\left.\left.\tau \in\right] 0, t\right]$ and $t \in\left[t_{0}, t_{1}\right]$. Then the function

$$
t \mapsto \xi_{t}(0)=\varphi(t)-f^{\prime}\left(u_{R}(t)\right) t
$$

is increasing.

Proof. If $t \mapsto \xi_{t}(0)$ is not increasing, then lines (2.5) cross each other in the time interval $\left.] 0, T\right]$. Hence, the solution $u$ has a (left contact) discontinuity $x=\psi(t)$ such that

$$
\varphi(\tau)<\psi(\tau)<\xi_{t_{1}}(\tau),
$$

Since the discontinuity at $x=\psi(t)$ can not be canceled [10, Theorem 2.3], and since it cannot intersect the line $x=\xi_{t_{1}}(\tau)$ due to the structure of minimal and maximal backward characteristics [10, Theorems 2.1 and 2.2], it turns out that $x=\psi(t)$ interacts with $\varphi(t)$ at a time $\bar{t} \leq t_{1}$. This can not occur, otherwise $\varphi(t)$ would not be a left contact discontinuity for $t \geq \bar{t}$.

Lemma 2.4 from [10] states that any left contact discontinuity associated to a BV solution $u$ of (1.1a) propagates along a strictly convex curve. This fact translates into a geometric condition on the lines $\eta_{x}$ : If $v$ is admissible and the candidate backward characteristic lines $\eta_{x}$ do not reach $t=0$ without crossing each other, then their tangent envelope must necessarily be a strictly convex curve. The following Proposition puts forward conditions on $v$ which ensure that this desirable geometry is realized.

Proposition 2.3. Let $I \subseteq \mathbb{R}$ be an interval, and $v \in \mathcal{C}^{0}(I)$ be bounded. Assume that the function $I \ni x \mapsto f^{\prime}(v(x))$ is differentiable with $D_{x} f^{\prime}(v(x))>0$ and $D^{+}\left(D_{x} f^{\prime}(v(x))\right)>0$ for all $x \in I$. Then, the tangent envelope of the lines $t \mapsto \eta_{x}(t)$ defined at (1.14c) for $x \in I$ is a strictly convex $C^{1}$ curve $x=\varphi(\tau)$, defined in a suitable interval $\left.\left.\left[t_{0}, t_{1}\right] \subseteq\right]-\infty, T\right]$. Moreover, if $D_{x} f^{\prime}(v(x)) \geq 1 / T$ for all $x \in I$ then the interval $\left[t_{0}, t_{1}\right]$ is contained in $[0, T]$.

Proof. Observe that, if $\varphi$ exists, then for any $\tau \in\left[t_{0}, t_{1}\right]$ there exists $x(\tau) \in I$ such that

$$
\varphi(\tau)=x(\tau)+f^{\prime}(v(x(\tau)))(\tau-T) \quad \text { and } \quad \varphi^{\prime}(\tau)=f^{\prime}(v(x(\tau))) .
$$


Moreover, since we want $\varphi$ to be strictly convex, the function $\tau \mapsto x(\tau)$ has to be strictly increasing, and has a strictly increasing inverse, say $I \ni x \mapsto t(x)$. Let us compute $t(x)$ in order to express $\varphi$ in terms of the $x$-variable. For any $x_{1}, x_{2} \in I$ the time at which the lines $\eta_{x_{1}}$ and $\eta_{x_{2}}$ intersect is given by

$$
t\left(x_{1}, x_{2}\right)=T-\frac{x_{2}-x_{1}}{f^{\prime}\left(v\left(x_{2}\right)\right)-f^{\prime}\left(v\left(x_{1}\right)\right)}<T,
$$

where we have used that $D_{x} f^{\prime}(v(x))>0$ for all $x \in I$. It follows that

$$
t\left(x_{1}\right)=\lim _{x_{2} \rightarrow x_{1}^{-}} t\left(x_{1}, x_{2}\right)=T-\frac{1}{D_{x} f^{\prime}\left(v\left(x_{1}\right)\right)} .
$$

Hence

$$
\varphi(t(x))=x+f^{\prime}(v(x))(t(x)-T)=x-\frac{f^{\prime}(v(x))}{D_{x} f^{\prime}(v(x))} .
$$

By construction, being $D^{+}\left(D_{x} f^{\prime}(v(x))\right)>0$ for all $x \in I, t(x)$ turns out to be strictly increasing. Moreover, as $\tau \mapsto x(\tau)$ is the inverse of $x \mapsto t(x)$ equation (2.6) implies that for a.e. $t$

$$
\begin{aligned}
\varphi^{\prime}(\tau) & =x^{\prime}(\tau)+D_{x} f^{\prime}(v(x(\tau))) x^{\prime}(\tau)(\tau-T)+f^{\prime}(v(x(\tau))) \\
& =x^{\prime}(\tau)\left[1+D_{x} f^{\prime}(v(x(\tau)))(\tau-T)\right]+f^{\prime}(v(x(\tau)))=f^{\prime}(v(x(\tau))) .
\end{aligned}
$$

Now one can easily conclude that the map $\varphi$ at (2.7) fulfills the desired properties.

Remark 2.1. Observe that, having in mind an admissible profile $v$, Proposition 2.3 and equation (2.6) imply that, if $v$ suffers from a left contact discontinuity at $x=\bar{x}$ then

$$
D_{x} f^{\prime}(v(x))=-\frac{1}{t(x)-T} \rightarrow+\infty \quad \text { as } x \rightarrow \bar{x}^{-} .
$$

This behaviour is forecast by the general theory on non-convex conservation law, see [16, Theorem 3.1].

Assume that an attainable profile $v$ suffers form a jump discontinuity at point $x=\bar{x}$ between the states $v(\bar{x})$ and $v\left(\bar{x}^{+}\right)=(v(\bar{x}))^{\sharp}$, and satisfies the hypothesis of Proposition 2.3 in a left neighbourhood $I$ of $\bar{x}$. In this framework we can define a curve $\tau \mapsto \varphi(\tau)$ and conclude that any solution $u$ of (1.1a) attaining the profile $v$ at time $T$ needs to suffer from a left contact discontinuity along the curve $\varphi$. It is easy and extremely important to notice that the values of $u$ on the two sides of $\varphi$ are completely determined as $u(\tau, \varphi(\tau))=v(x(\tau))$ and $u\left(\tau, \varphi(\tau)^{+}\right)=(v(x(\tau)))^{\sharp}$, the latter being defined as at (1.7) with $v=v(x(\tau))$. Using the inverse function $x \mapsto t(x)$ introduced in the proof of Proposition 2.3 and writing $v^{\sharp}(x)$ for $(v(x))^{\sharp}$, we can adapt the expression (2.5) to describe the candidate maximal backward characteristic from $(\tau, x(\tau))=(t(x), \varphi(t(x)))$ in terms of the profile $v$ only, see figure 1 ,

$$
\xi_{t(x)}(\tau)=\varphi(t(x))+f^{\prime}\left(v^{\sharp}(x)\right)(\tau-t(x)) .
$$

For future reference, we notice here that equations (2.6) and (2.7) imply 


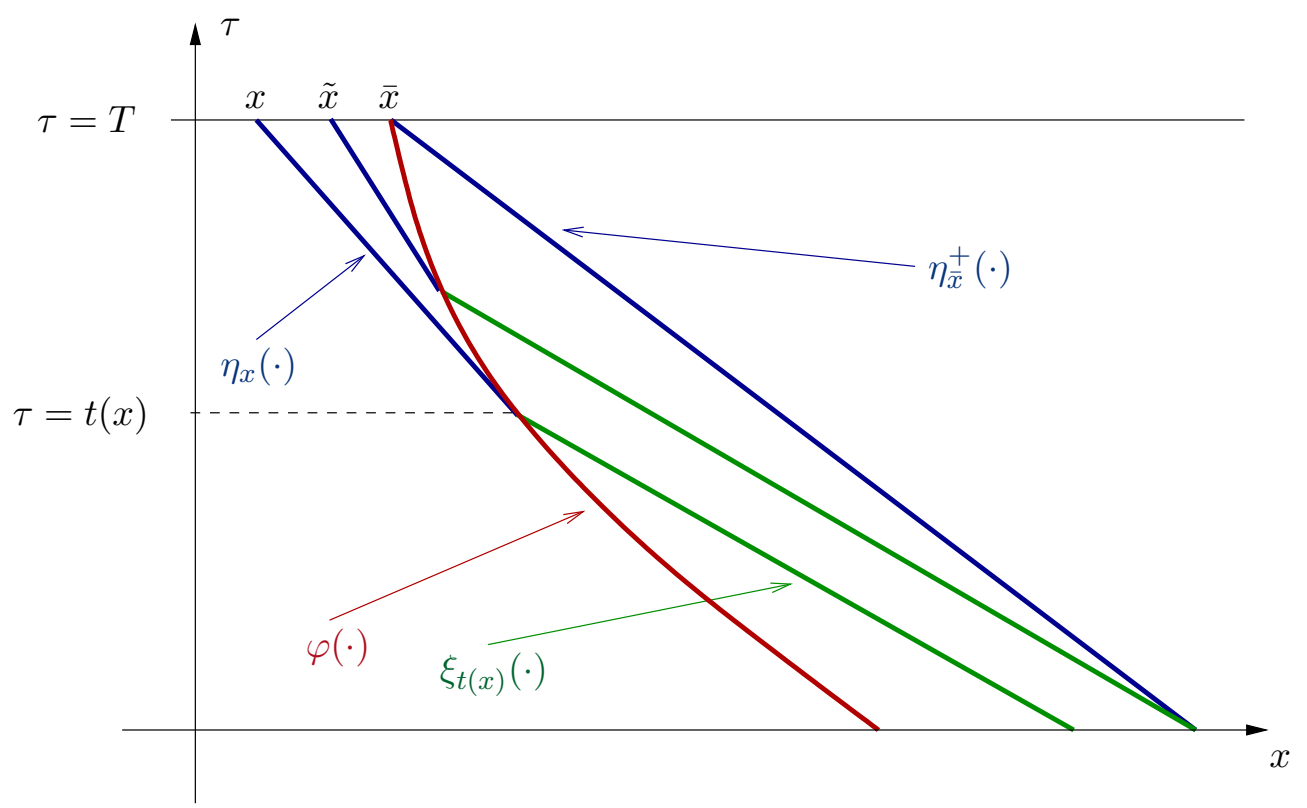

Figure 1: The lines $\eta_{x}$ and $\xi_{t(x)}$

$$
\begin{aligned}
\xi_{t(x)}(0)=\varphi(t(x))-f^{\prime}\left(v^{\sharp}(x)\right) t(x) & =x-\frac{f^{\prime}(v(x))}{D_{x} f^{\prime}(v(x))}-f^{\prime}\left(v^{\sharp}(x)\right)\left[T-\frac{1}{D_{x} f^{\prime}(v(x))}\right] \\
& =x+\frac{f^{\prime}\left(v^{\sharp}(x)\right)-f^{\prime}(v(x))}{D_{x} f^{\prime}(v(x))}-f^{\prime}\left(v^{\sharp}(x)\right) T .
\end{aligned}
$$

The proof of the following proposition is omitted, since it follows easily from Propositions 2.2 and 2.3 .

Proposition 2.4. Assume that $v$ is a measurable, left continuous, bounded attainable profile, with finite total variation. Assume that $v$ suffers from a left contact discontinuity at $x=\bar{x}$ with right state $v_{R}$. Assume that there exists an open interval $\left.\left.I \subset\right]-\infty, \bar{x}\right]$ such that

1. $\sup I=\bar{x}$;

2. $v \in \mathcal{C}^{0}(I)$;

3. the function $I \ni x \mapsto f^{\prime}(v(x))$ is differentiable and $D_{x} f^{\prime}(v(x)), D^{+}\left(D_{x} f^{\prime}(v(x))\right)>0$ for any $x \in I$.

Moreover, let $\eta_{\bar{x}}^{+}(\cdot)$ be defined as at (1.14b) with $x=\bar{x}, \xi_{t(x)}(\cdot)$ be defined as at $(2.8)$, and let

$$
\begin{aligned}
\tilde{x} & =\sup \left\{x \in I: \xi_{t(x)}(0) \leq \eta_{\bar{x}}^{+}(0)\right\} \\
& =\sup \left\{x \in I: x+\frac{f^{\prime}\left(v^{\sharp}(x)\right)-f^{\prime}(v(x))}{D_{x} f^{\prime}(v(x))}-f^{\prime}\left(v^{\sharp}(x)\right) T \leq \bar{x}-f^{\prime}\left(v_{R}\right) T\right\}
\end{aligned}
$$


(see figure 1). Then, the function $x \mapsto \xi_{t(x)}(0)$ is increasing in $I \backslash \tilde{x}, \bar{x}[$.

Remark 2.2. In the framework of Proposition 2.4, some properties of the attainable profile $v$ in the interval $x \in] \tilde{x}, \bar{x}$ [ will be discussed in Section 5. We point out that Proposition 2.4 states that, if a second left contact appears at the left of $x=\varphi(t)$, then it is necessary located at the left of $x=\eta_{\bar{x}}^{+}(t)$.

\section{Sufficient conditions for attainability}

We start by formulating the main result of this paper, which provides a partial description of the set $\mathcal{U}_{T}$. The meaning of the most technical hypotheses is explained in the three Remarks following the statement.

Theorem 2. Let $v \in B V(\mathbb{R})$ be a left continuous function with a nowhere dense set of points of jump discontinuities. Let $\left[\alpha_{0}, \beta_{0}\right]$ be the support of $v$ and let $\alpha$ be defined as at (1.13). Assume that the following conditions hold.

1. $v$ fulfills conditions 1-4 of Theorem 1 .

2. We consider the partition of $\left[\alpha_{0}, \beta_{0}\right]$ into maximal subintervals in which $v$ is continuous. For any of these maximal subintervals, say I, the following conditions are fullfilled. Define

$$
J \doteq\left\{x \in I: D_{x} f^{\prime}(v(x))>1 / T\right\}
$$

Assume that either $J$ is an empty set, or that the following conditions hold

(a) $J$ is a subinterval of $I$ and $\sup J=\sup I=\bar{x}$ is a point of jump of $v$. We let $v_{L}$ and $v_{R}$ be the left and right states of $v$ at $\bar{x}$, respectively.

(b) $v_{L} \doteq v(\bar{x})$ is different from zero.

(c) Let $v^{\sharp}(x)$ be $(v(x))^{\sharp}$ as it is defined by the formula (1.7). We have

$$
\begin{gathered}
D^{+}\left(D_{x} f^{\prime}(v(x))\right)>0 \quad \forall x \in J ; \\
D^{+}\left[x+\frac{f^{\prime}\left(v^{\sharp}(x)\right)-f^{\prime}(v(x))}{D_{x} f^{\prime}(v(x))}-f^{\prime}\left(v^{\sharp}(x)\right) T\right] \geq 0 \quad \forall x \in J .
\end{gathered}
$$

(d) Let $\left(v_{L}^{\sharp}\right)^{\sharp}$ be as $v^{\sharp}$ in (1.7) with $v=v_{L}^{\sharp}$. If $v_{R} \in I\left(v_{L},\left(v_{L}^{\sharp}\right)^{\sharp}\right)$, there holds

$$
\frac{\left(v_{L}^{\sharp}-v_{R}\right)\left[f^{\prime}\left(v_{L}\right)-f^{\prime}\left(v_{L}^{\sharp}\right)\right]}{f\left(v_{R}\right)-f\left(v_{L}\right)-\left(v_{R}-v_{L}\right) f^{\prime}\left(v_{L}\right)}\left[T-\frac{1}{D_{x}^{-} f^{\prime}(v(\bar{x}))}\right] \leq T \text {. }
$$

Then, $v$ is an attainable profile, and hence there exists $u_{c} \in L^{\infty}(a, b)$ such that the weak entropy solution $u=u(t, x)$ to (1.1a)-(1.1b) satisfies $u(T, \cdot)=v$. 
Remark 3.1. If $J$ is empty all the candidate backward characteristics $\eta_{x}$ for $x \in I$ are classical characteristics and reach $t=0$ without crossing each other. The backward reconstruction of $v$ in this case is known, as it only contains genuine shocks and rarefaction waves, see also Proposition 2.1. When $J$ is not empty the backward reconstruction of $v$ contains a left contact discontinuity.

Conditions $2 \mathrm{a}$ and $2 \mathrm{~b}$ are actually necessary conditions. In particular, $2 \mathrm{~b}$ is related to a property stated by Dafermos in [10, Theorem 2.2]. Indeed, if $v_{L}=0$, the minimal backward characteristic from $(t, \bar{x})$ is the straight line $x=\bar{x}$ and no crossing of characteristics occurs. This is in contradiction with the hypothesis that $J$ is not empty.

Remark 3.2. Condition $2 \mathrm{c}$ tells us that the family of candidate backward characteristics generated from the points $(T, x)$, for $x \in J$, admits a convex tangent envelope $\tau \mapsto \varphi(\tau)$ and that the candidate maximal backward characteristics $\xi_{t(x)}$ defined as at (2.8) do not cross at positive times. Indeed, condition (3.3) means that the function $x \mapsto \xi_{t(x)}(0)$, see (2.9), is increasing for all $x \in J$. In other words this condition excludes the occurrence of nesting phenomena. We postpone to Section 5 the analysis of some situations in which nesting takes place.

Remark 3.3. In the setting of condition $2 \mathrm{~d}$, namely if $v_{R} \in I\left(v_{L},\left(v_{L}^{\sharp}\right)^{\sharp}\right)$, the discontinuity at $x=\bar{x}$ in the target profile $v$ is not a left contact but a shock. However, given the structure of the candidate backward characteristics ( $J$ is non empty), we expect that in any solution $u$ of (1.1a) attaining $v$ at time $T$ such a shock origins from an interaction at $\tau<T$ involving a left contact discontinuity. Therefore we stress that $D_{x}^{-} f^{\prime}(v(\bar{x}))$ is finite, as we are not in the same setting as Remark 2.1, and being $D_{x}^{-} f^{\prime}(v(\bar{x})) \geq 1 / T$, condition (3.4) implies

$$
\frac{\left(v_{L}^{\sharp}-v_{R}\right)\left[f^{\prime}\left(v_{L}\right)-f^{\prime}\left(v_{L}^{\sharp}\right)\right]}{f\left(v_{R}\right)-f\left(v_{L}\right)-\left(v_{R}-v_{L}\right) f^{\prime}\left(v_{L}\right)} \geq 1 .
$$

The precise form of inequality (3.4) comes from the construction in the proof of Lemma 3.3 below.

\subsection{The building blocks of our construction}

This section is devoted to the detailed analysis of three basic cases in which the existence of a control function $u_{c}$ can be easily proved. These cases are used as building blocks in the proof of Theorem 2. Indeed, under the assumptions of Theorem 2 the nesting effect can not be observed, see Remark 3.2. Therefore, it is possible to split the support of $v$ into subintervals $I_{k}$, show the existence of controls $u_{c}^{k}$ which allow to attain the truncated functions $v_{\left.\right|_{I_{k}}}$, and finally "glue" together the functions $u_{c}^{k}$ to obtain a control $u_{c}$ such that the weak entropy solution $u=u(t, x)$ to $(1.1 \mathrm{a})-(1.1 \mathrm{~b})$ satisfies $u(T, \cdot)=v$. This argument works because the domain of dependence of $u(T, \cdot)$ on each interval $I_{k}$, in absence of nesting, is delimited by the maximal backward generalized characteristics from $t=T, x=\inf I_{k}$ and $t=T, x=\sup I_{k}$.

For the reader's convenience, we fix here the notation and the setting which will be used all through this section. Roughly speaking, we isolate one of the intervals $I_{k}$. 
(V) Let $v \in \mathrm{BV}(\mathbb{R})$ satisfy all the hypothesis of Theorem 2 together with the following stronger assumptions. Let $v$ be left continuous, compactly supported on $] \alpha_{0}, \beta_{0}[$ and suffering of a single jump discontinuity at the point $x=\bar{x}$, connecting the values $v(\bar{x})=v_{L}$ and $v_{R}$. For definiteness we assume that $v_{L}<0$ (the case $v_{L}>0$ is symmetric) and that $v(x)=v_{R}$ for all $x$ in $] \bar{x}, \beta_{0}\left[{ }^{2}\right.$. We assume, as prescribed by condition 1 of Theorem 2 , that the jump $\left(v_{L}, v_{R}\right)$ is admissible in the sense of condition 4 of Theorem 1 . Using the same notation as in Theorem 2 we set $I \doteq] \alpha_{0}, \bar{x}$ [ and $J \doteq\left\{x \in I: D_{x} f^{\prime}(v(x))>1 / T\right\}$. If $J$ is not empty, we define $\hat{x} \doteq \inf J$, while $\sup J$ has to be $\bar{x}$ according to condition 2a of Theorem 2.

We recall that the (candidate) backward characteristic lines $\eta_{x}^{+}, \eta_{x}^{-}$and $\eta_{x}$ have been introduced at (1.14). Whenever $J$ is non empty, condition (3.2) ensures that the tangent envelope of the lines $\tau \mapsto \eta_{x}(\tau)$, for $x \in J$, is a convex curve $x=\varphi(\tau)$, defined for $\tau \in\left[t_{0}, t_{1}\right]$ with $t_{1}=t(\bar{x})$ (see $(2.6))$ given by

$$
\begin{aligned}
t_{1} & =\sup \left\{t \leq T: \eta_{x}(t)=\eta_{\bar{x}}(t), x \in J, x<\bar{x}\right\} \\
& =\sup _{\substack{x \in I \\
x<\bar{x}}}\left\{T-\frac{x-\bar{x}}{f^{\prime}(v(x))-f^{\prime}\left(v_{L}\right)}\right\}=T-\frac{1}{D_{x}^{-} f^{\prime}(v(\bar{x}))},
\end{aligned}
$$

and $t_{0}$ given by

$$
t_{0}=\max \left\{0, t \text { s.t. } \varphi^{\prime}(t)=f^{\prime}(v(\hat{x}))\right\} .
$$

The presentation articulates into three lemmas, focusing respectively on

Case 1: The profile $v$ suffers from a shock discontinuity at $x=\bar{x}$ and the candidate backward characteristics $\eta_{x}$, for $x \in I$ do not cross in the time interval $\left.] 0, T\right]$.

Case 2: The set $J$ is not empty, therefore the candidate backward characteristics $\eta_{x}$, for $x \in J$ cross in positive time. The state $v_{R}$ does not belong to the open interval with extrema $v_{L}^{\sharp}$ and $\left(v_{L}^{\sharp}\right)^{\sharp}$. This means that the profile $v$ suffers either from a left contact discontinuity at $x=\bar{x}$, i.e. $v_{R}=\left(v_{L}\right)^{\sharp}$, or the discontinuity of $v$ at $\bar{x}$ can be solved backward as an interaction occurring at time $\tau=T$ and involving a left contact discontinuity.

Case 3: The set $J$ is not empty but the state $v_{R}$ lies in between $v_{L}$ and $\left(v_{L}^{\sharp}\right)^{\sharp}$. Therefore, on the one hand the jump of $v$ at $\bar{x}$ can only be interpreted as a shock, on the other hand the crossing of backward characteristics force the presence of a left contact discontinuity in any entropy weak solution $u$ such that $u(T)=v$. This construction is the most intricate.

We stress that in Cases 1, 2 and 3 we can explicitly construct a control function $u_{c}$.

Lemma 3.1 (Case 1). Assume that $v$ is as in (V) and that $J$ is empty. Then one can explicitly provide an initial condition $u_{1} \in L^{\infty}\left(\eta_{\alpha_{0}}^{+}(0), \eta_{\beta_{0}}^{-}(0)\right)$, and a weak entropy solution u (see (3.8) below) to

$$
\left\{\begin{array}{l}
\partial_{t} u+\partial_{x} f(u)=0, \\
u(0, x)=u_{1}(x),
\end{array} \quad 0 \leq t \leq T, \eta_{\alpha_{0}}^{+}(t)<x<\eta_{\beta_{0}}^{-}(t),\right.
$$

\footnotetext{
${ }^{2}$ Please notice that the constructions we perform are essentially the same under the (slightly) weaker hypothesis that the set $J_{x>\bar{x}} \doteq\{x \in] \bar{x}, \beta_{0}\left[: D_{x} f^{\prime}(v(x))>1 / T\right\}$ is empty.
} 
such that $u(T, x)=v(x)$ a.e. in $] \alpha_{0}, \beta_{0}[$.

Proof. In this case we can explicitly construct a backward solution $u$, hence a control $u_{1}$, in the following classical way. The shock wave joining the states $v_{L}$ and $v_{R}$ propagates along the line

$$
\mathfrak{s}(\tau)=\bar{x}+\sigma\left(v_{L}, v_{R}\right)(\tau-T)
$$

where

$$
\sigma\left(v_{L}, v_{R}\right)=\frac{f\left(v_{R}\right)-f\left(v_{L}\right)}{v_{R}-v_{L}}
$$

is the Rankine-Hugoniot speed of the jump $\left(v_{L}, v_{R}\right)$. Then

$$
u(\tau, y)=\left\{\begin{array}{lll}
v(x) & \text { if } \left.\quad \eta_{\alpha_{0}}^{+}(\tau)<y=\eta_{x}(\tau) \leq \eta_{\bar{x}}^{-}(\tau), \tau \in\right] 0, T[ \\
v_{L} & \text { if } \quad \eta_{\bar{x}}(\tau)<y \leq \mathfrak{s}(\tau), \\
v_{R} & \text { if } \quad \mathfrak{s}(\tau)<y<\eta_{\beta_{0}}^{-}(\tau),
\end{array}\right.
$$

that is obtained just following up to $t=0$ the backward characteristics. Please notice that in this case the backward reconstruction is far from being unique.

Now we turn our attention to the most interesting case $J \neq \emptyset$.

Lemma 3.2 (Case 2). Assume that $v$ is as in (V), $J$ is not empty and $v_{R}$ does not belong to the open interval of extrema $v_{L}^{\sharp}$ and $\left(v_{L}^{\sharp}\right)^{\sharp}$. Then one can explicitly provide an initial condition $u_{2} \in L^{\infty}\left(\eta_{\alpha_{0}}^{+}(0), \eta_{\beta_{0}}^{-}(0)\right)$, and a weak entropy solution $u$ (see (3.9)-(3.10c) below) to

$$
\left\{\begin{array}{l}
\partial_{t} u+\partial_{x} f(u)=0, \\
u(0, x)=u_{2}(x),
\end{array} \quad 0 \leq t \leq T, \eta_{\alpha_{0}}^{+}(t)<x<\eta_{\beta_{0}}^{-}(t),\right.
$$

such that $u(T, x)=v(x)$ a.e. in $] \alpha_{0}, \beta_{0}[$.

Proof. As we assume that $v_{L}<0$, either $0<v_{L}^{\sharp} \leq v_{R}$ or $v_{R} \leq\left(v_{L}^{\sharp}\right)^{\sharp}<0$. If $v_{R}=v_{L}^{\sharp}$ the discontinuity of $v$ at $x=\bar{x}$ is a left contact discontinuity. If $v_{R}=\left(v_{L}^{\sharp}\right)^{\sharp}$ the discontinuity of $v$ at $x=\bar{x}$ can be solved backward as an interaction occurring at time $T$ between the left contact discontinuities from $v_{L}$ to $v_{L}^{\sharp}$ and from $v_{L}^{\sharp}$ to $\left(v_{L}^{\sharp}\right)^{\sharp}$. In all other cases the discontinuity of $v$ at $x=\bar{x}$ can be solved backward as an interaction occurring at time $T$ between the left contact discontinuity from $v_{L}$ to $v_{L}^{\sharp}$ and a shock joining $v_{L}^{\sharp}$ to $v_{R}$, see figure 2 .

Once we fixed the structure of a backward resolution of the discontinuity, the construction of a control becomes routine. Indeed, assumption (3.3) ensures that nesting does not occur, therefore the backward reconstruction of the solution can be done up to $t=0$ without the appearance of unexpected waves.

In order to keep our presentation as light as possible, we limit the discussion to the case $v_{R} \notin\left\{v_{L}^{\sharp},\left(v_{L}^{\sharp}\right)^{\sharp}\right\}$, as the two other cases can be easily to inferred from this one.

Call $\mathfrak{s}$ the line along which the shock joining $v_{L}^{\sharp}$ to $v_{R}$ propagates in the backward solution

$$
\mathfrak{s}(\tau)=\bar{x}+\sigma\left(v_{L}^{\sharp}, v_{R}\right)(\tau-T),
$$


where $\sigma\left(v_{L}^{\sharp}, v_{R}\right)$ is the Rankine-Hugoniot speed of the jump $\left(v_{L}^{\sharp}, v_{R}\right)$. We fix the values of $t_{0}$ and $t_{1}$ as in (3.6) and(3.5). A backward solution to (1.1a) with datum $u(T, x)=v(x)$ takes the form

$$
u(\tau, y)=\left\{\begin{array}{lll}
v(x) & \text { if } & \left(\tau, y=\eta_{x}(\tau)\right) \in \Omega_{1}, \\
(v(x))^{\sharp} & \text { if } \quad\left(\tau, y=\xi_{t(x)}(\tau)\right) \in \Omega_{2}, \\
v_{L}^{\sharp} & \text { if } \quad(\tau, y) \in \Omega_{3}, \\
v_{R} & \text { if } \quad y \in] \mathfrak{s}(\tau), \eta_{\beta_{0}}^{-}[, \tau \in] 0, T[.
\end{array}\right.
$$

where the regions $\Omega_{i}$, for $i=1,2,3$, are defined as follows.

$$
\begin{aligned}
& \Omega_{1}=\{\left.\left.\left.\left.\left.\left.(\tau, y) \in] t_{1}, T[\times] \eta_{\alpha_{0}}^{+}(\tau), \eta_{\bar{x}}^{-}(\tau)\right]\right\} \cup\{(\tau, y) \in] t_{0}, t_{1}\right] \times\right] \eta_{\alpha_{0}}^{+}(\tau), \varphi(\tau)\right]\right\} \\
&\left.\left.\cup\{(\tau, y) \in] 0, t_{0}[\times] \eta_{\alpha_{0}}^{+}(\tau), \eta_{\hat{x}}(\tau)\right]\right\}, \\
&\left.\left.\left.\left.\left.\left.\Omega_{2}=\{(\tau, y) \in] t_{0}, t_{1}[\times] \varphi(\tau)^{+}, \xi_{t_{1}}(\tau)\right]\right\} \cup\{(\tau, y) \in] 0, t_{0}\right] \times\right] \eta_{\hat{x}}(\tau), \xi_{t_{1}}(\tau)\right]\right\}, \\
&\left.\left.\left.\left.\left.\left.\Omega_{3}=\{(\tau, y) \in] t_{1}, T[\times] \eta_{\bar{x}}^{-}(\tau), \mathfrak{s}(\tau)\right]\right\} \cup\{(\tau, y) \in] 0, t_{1}\right] \times\right] \xi_{t_{1}}(\tau), \mathfrak{s}(\tau)\right]\right\} .
\end{aligned}
$$

Then $u_{2}$ can be constructed just following backwardly the backward characteristics up to $t=0$.

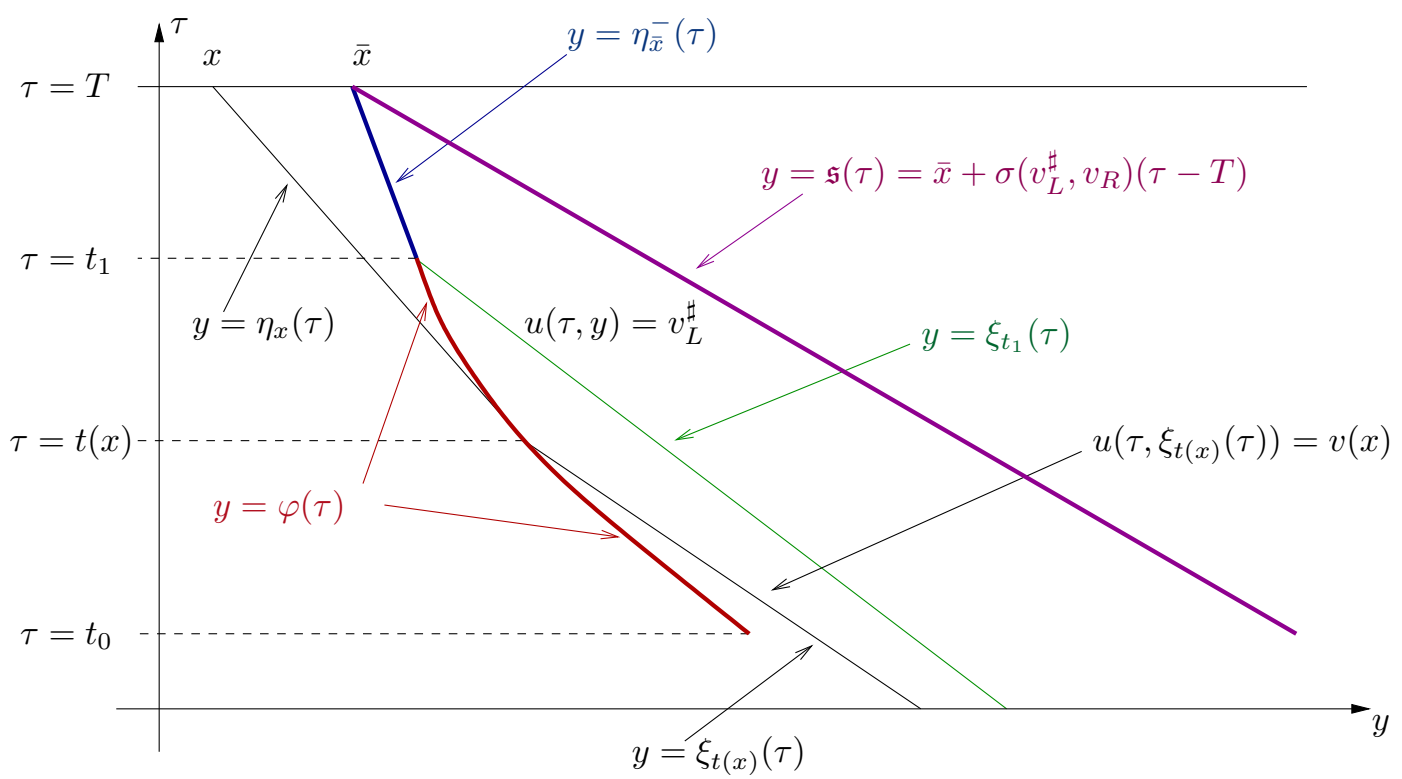

Figure 2: The function $u$ at (3.9)

Lemma 3.3 (Case 3$)$. Assume that $v$ is as in $(\mathbf{V}), J$ is not empty and $v_{R} \in I\left(v_{L},\left(v_{L}^{\sharp}\right)^{\sharp}\right)$. Then there exists an initial condition $u_{3} \in L^{\infty}\left(\eta_{\alpha_{0}}^{+}(0), \eta_{\beta_{0}}^{-}(0)\right)$, such that the weak entropy solution u to

$$
\left\{\begin{array}{l}
\partial_{t} u+\partial_{x} f(u)=0, \\
u(0, x)=u_{3}(x),
\end{array} \quad 0 \leq t \leq T, \eta_{\alpha_{0}}^{+}(t)<x<\eta_{\beta_{0}}^{-}(t),\right.
$$


satisfies $u(T, x)=v(x)$ a.e. in $] \alpha_{0}, \beta_{0}[$.

Remark 3.4. Regarding Lemma 3.3, we provide at the end of the proof an explicit formulation of the initial datum $u_{3}$ and of the solution $u$ to (3.11) attaining $v$ at time $T$.

Proof. Our first step in this proof is to find a wave pattern which is compatible with the information we have on the profile $v$. Here the jump of $v$ at $\bar{x}$ can only be interpreted as a shock, and, since $J \neq \emptyset$, we can argue the presence of a left contact discontinuity $\varphi$ in any entropy weak solution $u$ such that $u(T)=v$. It is well known, see [10, Theorem 2.3], that once a left contact discontinuity appears in a weak entropy solution of (1.1a), it can not disappear but can interact with other waves. Therefore we conclude that the shock jump in $v$ at $x=\bar{x}$ corresponds to a shock wave $\mathfrak{s}_{3}$ in $u$ originated from the interaction between $\varphi$ and another discontinuity $\mathfrak{s}_{0}$. The idea, roughly speaking, is the following. First, we use the values of $v$ for $x<\bar{x}$ to perform a partial backward reconstruction of the left contact discontinuity $\varphi$ and, in particular, find the time $t_{1}$ at which the candidate minimal backward characteristic from $(T, \bar{x})$ is tangent to $\varphi$, see equation (3.5). Second, we trace the maximal backward characteristic $\xi_{t_{1}}$ from $\left(t_{1}, \varphi\left(t_{1}\right)\right)$ and we call $x_{2}$ the point $\xi_{t_{1}}(0)$. We also trace the maximal backward characteristic $\eta_{\bar{x}}^{+}$from $(T, \bar{x})$ and we call $\left(t^{*}, x^{*}\right)$ the intersection between $\xi_{t_{1}}$ and $\eta_{\bar{x}}^{+}$

$$
\xi_{t_{1}}\left(t^{*}\right)=\eta_{\bar{x}}^{+}\left(t^{*}\right)=x^{*}
$$

The condition $t^{*} \leq 0$ turns out to be necessary for the attainability of $v$ (see below).

Finally, we construct a one parameter family of initial conditions $\left(w_{\gamma}^{0}\right)_{\gamma \in\left[t^{*}, 0\right]}$ such that

1. the function $\gamma \mapsto w_{\gamma}^{0}$ is continuous;

2. all corresponding forward solutions $w_{\gamma}$ to (1.1a) have the desired wave pattern (incoming $\varphi$ and $\mathfrak{s}_{0}$, interaction, outgoing $\left.\mathfrak{s}_{3}\right)$;

3. there exists a value $\gamma^{*} \in\left[t^{*}, 0\right]$ such that the solution $w_{\gamma^{*}}$ associated to $w_{\gamma^{*}}^{0}$ attains exactly the profile $v$ at time $T$.

The rigorous presentation of the proof divides into several steps.

Step 1: Partial backward reconstruction. We consider the case $\left(v_{L}^{\sharp}\right)^{\sharp}<v_{R}<v_{L}<0$. With exactly the same notation as in the proof of Lemma 3.2 we use the method of characteristics to reconstruct the solution backward in the regions $\Omega_{1}$ and $\Omega_{3}$, see (3.10a), (3.10c). We call

$$
\begin{aligned}
& x_{1} \doteq \eta_{\bar{x}}^{-}\left(t_{1}\right)=\bar{x}+f^{\prime}\left(v_{L}\right)\left(t_{1}-T\right), \\
& x_{2} \doteq \xi_{t_{1}}(0)=x_{1}-f^{\prime}\left(v_{L}^{\sharp}\right) t_{1} .
\end{aligned}
$$


Step 2: The Riemann problem with data $\left(v_{L}^{\sharp}, \boldsymbol{v}_{\boldsymbol{R}}\right)$. In this step we introduce some notations and describe the self similar solution to a Riemann problem with data $\left(v_{L}^{\sharp}, v_{R}\right)$ centered at $(t=\mathfrak{t}, x=\mathfrak{a})$

$$
\begin{cases}u_{t}+f(u)_{x}=0 & \text { for } t>\mathfrak{t}, \text { and } x \in \mathbb{R}, \\ u(\mathfrak{t}, x)= \begin{cases}v_{L}^{\sharp} & \text { if } x \leq \mathfrak{a}, \\ v_{R} & \text { if } x>\mathfrak{a} .\end{cases} \end{cases}
$$

Observe that our assumptions on $f$ and the choice $v_{L}<0$ imply $0<v_{R}^{b}<v_{L}^{\sharp}$. Therefore, the solution to the problem (3.15) consists of a centered rarefaction wave with left state $v_{L}^{\sharp}$ and right state $v_{R}^{b}$, followed by a left contact discontinuity joining $v_{R}^{b}$ to $v_{R}, \tau \mapsto \mathfrak{s}_{0}(\tau)$. More precisely, we have

$$
\begin{aligned}
\mathfrak{s}_{0}(\tau) & =\mathfrak{a}+f^{\prime}\left(v_{R}^{b}\right)(\tau-\mathfrak{t}) \\
& =\mathfrak{a}+\frac{f\left(v_{R}\right)-f\left(v_{R}^{b}\right)}{v_{R}-v_{R}^{b}}(\tau-\mathfrak{t}) \quad \tau \geq \mathfrak{t},
\end{aligned}
$$

We call $\mathfrak{r}=\mathfrak{r}(\tau, y)$ the rarefaction fan outcoming from $(\mathfrak{t}, \mathfrak{a})$ with left and right state $v_{L}^{\sharp}$ and $v_{R}^{b}$, respectively. It is described as the unique solution to

$$
f^{\prime}(\mathfrak{r}(\tau, y))=\frac{y-\mathfrak{a}}{\tau-\mathfrak{t}}, \quad \mathfrak{r}(\tau, y) \geq 0
$$

and $v_{R}^{b} \leq \mathfrak{r}(\tau, y)<v_{L}^{\sharp}$.

Observe that in order to lighten the notation, the dependence of $\mathfrak{r}$ and $\mathfrak{s}_{0}$ on $\mathfrak{t}, \mathfrak{a}$ is somehow hidden, nevertheless it is absolutely essential to keep it in mind throughout the proof.

Step 3: A one parameter family of initial conditions. Let $\gamma \in\left[t^{*}, 0\right]$ be fixed and set $\mathfrak{t}=\gamma, \mathfrak{a}=\xi_{t_{1}}(\gamma)$. We define the initial condition $w_{\gamma}^{0}$ as follows

$$
w_{\gamma}^{0}(y)=\left\{\begin{array}{lll}
v(x) & \text { if } & \left.\left.y=\eta_{x}(0) \in\right] \eta_{\alpha_{0}}^{+}(0), \eta_{\hat{x}}(0)\right], \\
(v(x))^{\sharp} & \text { if } & \left.\left.y=\xi_{t(x)}(0) \in\right] \eta_{\hat{x}}(0), x_{2}\right] \\
\mathfrak{r}(0, y) & \text { if } & \left.y \in] x_{2}, \mathfrak{s}_{0}(0)\right] \\
v_{R} & \text { if } & y>\mathfrak{s}_{0}(0) .
\end{array}\right.
$$

Observe that the value of $w_{\gamma}^{0}(y)$ for $y \leq x_{2}$ is fixed by following the backward characteristics from the assigned profile $v$ for all $\gamma \in\left[t^{*}, 0\right]$. As a consequence, the solution of the Cauchy problem (1.1a)-(3.17), $w_{\gamma}$, coincides with the backward reconstruction in regions $\Omega_{1}$ and $\Omega_{3}$. For $y>x_{2}, w_{\gamma}^{0}$ coincides with the self similar solution of the Riemann problem between the states $\left(v_{L}^{\sharp}, v_{R}\right)$ centered at $\left(\gamma, \xi_{t_{1}}(\gamma)\right)$. The solution $w_{\gamma}$ can be constructed by the method of characteristics until at time $\tau=t_{1}$ the rarefaction fan $\mathfrak{r}$ reaches the contact discontinuity $\varphi$ (which we obtained by backward reconstruction). 
Step 4: Forward construction of $\boldsymbol{w}_{\gamma}$ for $\boldsymbol{\tau}>\boldsymbol{t}_{\mathbf{1}}$. For $\tau>t_{1}$ the contact discontinuity $\varphi$ interacts with the rarefaction fan $\mathfrak{r}$. The result of this interaction is a contact discontinuity with left state $\mathfrak{r}^{b}\left(\tau, \mathfrak{s}_{1}(\tau)\right)$ and right state $\mathfrak{r}\left(\tau, \mathfrak{s}_{1}(\tau)\right)$, traveling along the curve $\tau \mapsto \mathfrak{s}_{1}(\tau)$, defined as the unique solution of the Cauchy problem

$$
\left\{\begin{array}{l}
\mathfrak{s}_{1}^{\prime}=f^{\prime}\left(\mathfrak{r}^{b}\left(\tau, \mathfrak{s}_{1}\right)\right) \\
\mathfrak{s}_{1}\left(t_{1}\right)=x_{1},
\end{array} \quad \tau \geq t_{1} .\right.
$$

Observe that, thanks to (1.10), we have

$$
\mathfrak{s}_{1}^{\prime}(\tau)=\frac{f\left(\mathfrak{r}^{b}\left(\tau, \mathfrak{s}_{1}(\tau)\right)\right)-f\left(\mathfrak{r}\left(\tau, \mathfrak{s}_{1}(\tau)\right)\right)}{\mathfrak{r}^{b}\left(\tau, \mathfrak{s}_{1}(\tau)\right)-\mathfrak{r}\left(\tau, \mathfrak{s}_{1}(\tau)\right)} .
$$

Geometrical considerations show that the curve $\mathfrak{s}_{1}$ approaches $\mathfrak{s}_{0}$ until $\mathfrak{r}$ attains the value $v_{R}^{b}$. Call $t_{3}$ the time at which the interaction takes place, i.e. $\mathfrak{s}_{1}\left(t_{3}\right)=\mathfrak{s}_{0}\left(t_{3}\right)$. Let

$$
x_{3} \doteq \mathfrak{s}_{1}\left(t_{3}\right)=\mathfrak{s}_{0}\left(t_{3}\right)=\xi_{t_{1}}(\gamma)+f^{\prime}\left(v_{R}^{b}\right) t_{3},
$$

and observe that, by construction,

$$
\eta_{\bar{x}}^{-}(\tau)<\mathfrak{s}_{1}(\tau)<\mathfrak{s}_{0}(\tau) \quad \forall t_{1}<\tau<t_{3}
$$

For future reference, we call $\Omega_{4}$ the region in the $t$-x plane delimited by $\xi_{t_{1}}, \mathfrak{s}_{0}$ and $\mathfrak{s}_{1}$

$$
\begin{aligned}
&\left.\Omega_{4}=\left\{(\tau, y) \in \mathbb{R}^{2}: \tau \in\right] \gamma, t_{1}\right], \xi_{t_{1}}(\tau)\left.<y \leq \mathfrak{s}_{0}(\tau)\right\} \cup \\
&\left.\left.\cup\left\{(\tau, y) \in \mathbb{R}^{2}: \tau \in\right] t_{1}, t_{3}\right], \mathfrak{s}_{1}(\tau)<y \leq \mathfrak{s}_{0}(\tau)\right\}
\end{aligned}
$$

The interaction between $\mathfrak{s}_{0}$ and $\mathfrak{s}_{1}$, taking place at $\left(t_{3}, x_{3}\right)$, generates a single outgoing shock discontinuity traveling along the curve $\tau \mapsto \mathfrak{s}_{3}(\tau)$. We use again the method of characteristics to precise the definition of $\mathfrak{s}_{3}$. For any $(\tau, y)$ such that $\tau \in\left[t_{1}, t_{3}\right], \eta_{\bar{x}}^{-}(\tau) \leq y \leq \mathfrak{s}_{1}(\tau)$ or $\tau>t_{3}$ and $\eta_{\bar{x}}^{-}(\tau) \leq y<x_{3}+f^{\prime}\left(v_{R}^{b}\right)\left(\tau-t_{3}\right)$, let $s=s(\tau, y) \in\left[t_{1}, t_{3}\right]$ be such that

$$
y=\mathfrak{s}_{1}(s)+\mathfrak{s}_{1}^{\prime}(s)(\tau-s),
$$

and define

$$
\mathfrak{q}(\tau, y)=\mathfrak{r}^{b}\left(s(\tau, y), \mathfrak{s}_{1}(s(\tau, y))\right) .
$$

In such a way $\mathfrak{q}=\mathfrak{q}(\tau, y)$ is a Lipschitz continuous solution to (1.1a) within its domain of definition. Then, we let $\tau \mapsto \mathfrak{s}_{3}(\tau)$ be the solution to the Cauchy problem

$$
\left\{\begin{array}{l}
\mathfrak{s}_{3}^{\prime}(\tau)=\frac{f\left(v_{R}\right)-f\left(\mathfrak{q}\left(\tau, \mathfrak{s}_{3}(\tau)\right)\right)}{v_{R}-\mathfrak{q}\left(\tau, \mathfrak{s}_{3}(\tau)\right)} \\
\mathfrak{s}_{3}\left(t_{3}\right)=x_{3}
\end{array}\right.
$$

so that $y=\mathfrak{s}_{3}(\tau)$ is a shock curve joining the left state $\mathfrak{q}\left(\tau, \mathfrak{s}_{3}(\tau)\right)$ to the right state $v_{R}$. 
The solution to (3.21) is defined starting from $\tau=t_{3}$ and until the point $\left(\tau, \mathfrak{s}_{3}(\tau)\right)$ reaches the line $\eta_{\bar{x}}^{-}$. This happens in finite time since the slope of $\eta_{\bar{x}}^{-}$is larger that $\mathfrak{s}_{3}^{\prime}(\tau)$ for all $\tau>t_{3}$. We call $\bar{t}$ the time at which the crossing takes place. This allows for the following definition

$$
\begin{aligned}
\left.\Omega_{5}=\left\{(\tau, y) \in \mathbb{R}^{2}: \tau \in\right] t_{1}, t_{3}\right], \eta_{\bar{x}}^{-}(\tau) & \left.<y<\mathfrak{s}_{1}(\tau)\right\} \cup \\
& \left.\left.\cup\left\{(\tau, y) \in \mathbb{R}^{2}: \tau \in\right] t_{3}, \bar{t}\right], \eta_{\bar{x}}^{-}(\tau)<y<\mathfrak{s}_{3}(\tau)\right\}
\end{aligned}
$$

The solution of the Cauchy problem (1.1a), (3.17), $w_{\gamma}$, is the piecewise Lipschitz continuous function defined as follows for $\tau \in] 0, \bar{t}]$, see figure 3 for the case $\gamma=0$,

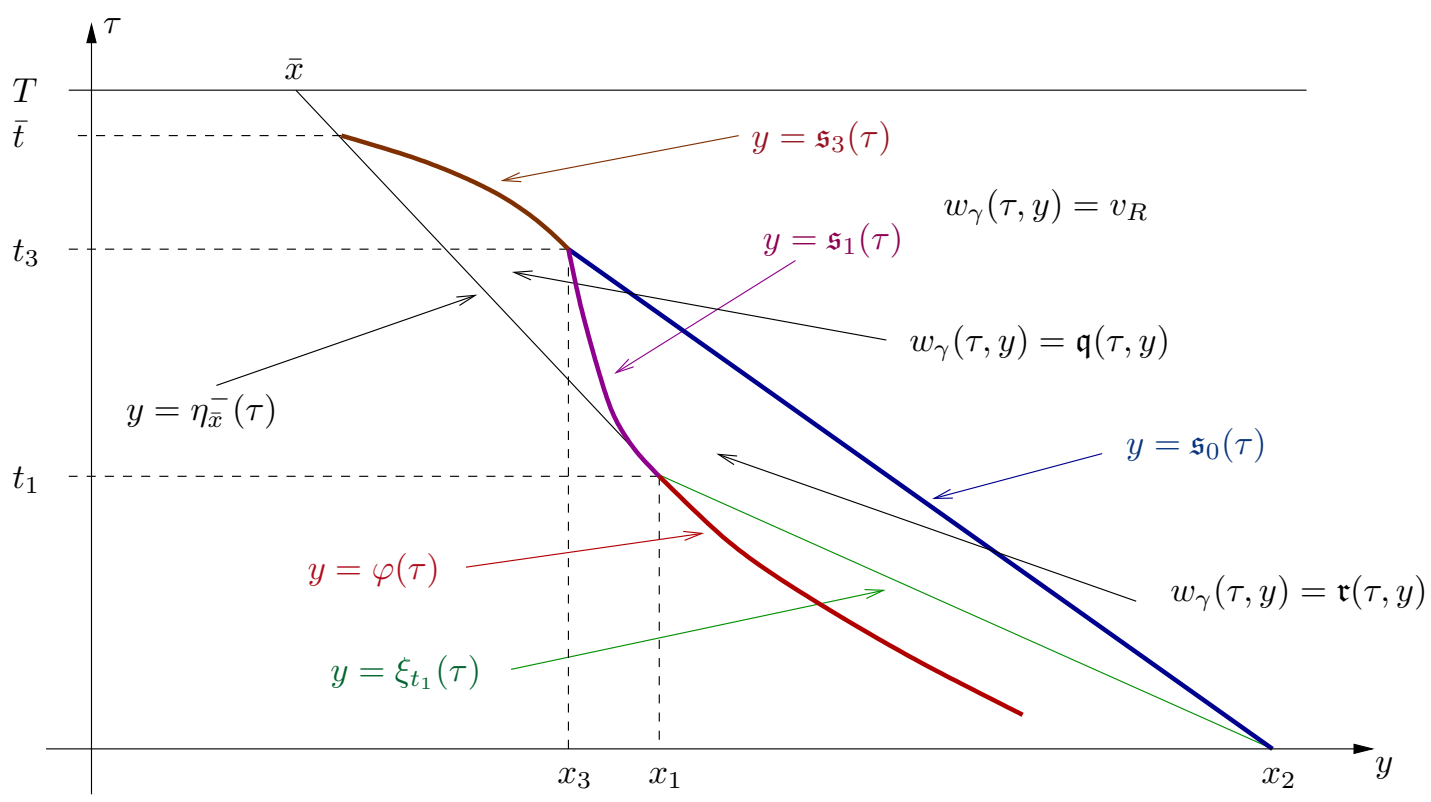

Figure 3: The case $\left(v_{L}^{\sharp}\right)^{\sharp}<v_{R}<v_{L}<0$

$$
w_{\gamma}(\tau, y)=\left\{\begin{array}{lll}
v(x) & \text { if } \quad\left(\tau, y=\eta_{x}(\tau)\right) \in \Omega_{1}, \\
(v(x))^{\sharp} & \text { if } \quad\left(\tau, y=\xi_{t(x)}(\tau)\right) \in \Omega_{2}, \\
\mathfrak{r}(\tau, y) & \text { if } \quad(\tau, y) \in \Omega_{4} \\
\mathfrak{q}(\tau, y) & \text { if } \quad(\tau, y) \in \Omega_{5} \\
v^{R} & \text { if } & \left.y \in] \mathfrak{s}_{0}(\tau), \eta_{\beta_{0}}^{-}(\tau)\right] \text { and } \tau \leq t_{3} \\
& \text { or } & \left.\left.\left.y \in] \mathfrak{s}_{3}(\tau), \eta_{\beta_{0}}^{-}(\tau)\right] \text { and } \tau \in\right] t_{3}, \bar{t}\right] .
\end{array}\right.
$$

The Lemma is proved if we can show that there exists a value of $\gamma$ for which $\bar{t}$ is exactly equal to $T$. Observe that, as the hypotheses of the Lemma exclude the occurrence of nesting, we are not allowed to consider initial conditions suffering from a jump discontinuity at a point $y \in] \eta_{\hat{x}}(0), x_{2}$ [. Therefore, when we consider $\gamma=0$ we obtain the solution of (1.1a) with the desired wave pattern (incoming $\varphi$ and $\mathfrak{s}_{0}$, interaction, outgoing $\mathfrak{s}_{3}$ ) in which the curves $\mathfrak{s}_{3}$ and $\eta_{\bar{x}}$ earlier than for any larger value of $\gamma$. 
Remark 3.5. We remark that a necessary condition toward the attainability of the profile $v$ is $t^{*}<0$, i.e.

$$
x_{2}<\eta_{\bar{x}}^{+}(0)=\bar{x}-f^{\prime}\left(v_{R}\right) T .
$$

Indeed, assume (3.24) does not hold (see figure 4). Since $f^{\prime}\left(v_{R}^{b}\right)>f^{\prime}\left(v_{R}\right)$, we get

$$
x_{3}>\bar{x}+f^{\prime}\left(v_{R}\right)\left(t_{3}-T\right) .
$$

Notice that $\mathfrak{s}_{3}^{\prime}(\tau)>f^{\prime}\left(v_{R}\right)$ for any $\tau$ due to the admissibility conditions for shock discontinuities, and that $v_{R}$ is the right state of $\mathfrak{s}_{3}$. Due to $(3.25)$ we have $\mathfrak{s}_{3}(T)>\bar{x}$. Hence, $\mathfrak{s}_{3}$ and $\eta_{\bar{x}}$ do not interact in the time interval $\left[t_{3}, T\right]$, contrary to the fact that $\bar{t} \leq T$. A similar argument shows that if $\gamma=t^{*}$ then for sure $\bar{t}>T$.

The next step in this proof shows that if our original target profile satisfies the hypothesis (3.4) of Theorem 2 then in $w_{\gamma=0}, \mathfrak{s}_{3}(\bar{t})=\eta_{\bar{x}}(\bar{t})$ at time $\bar{t}<T$.

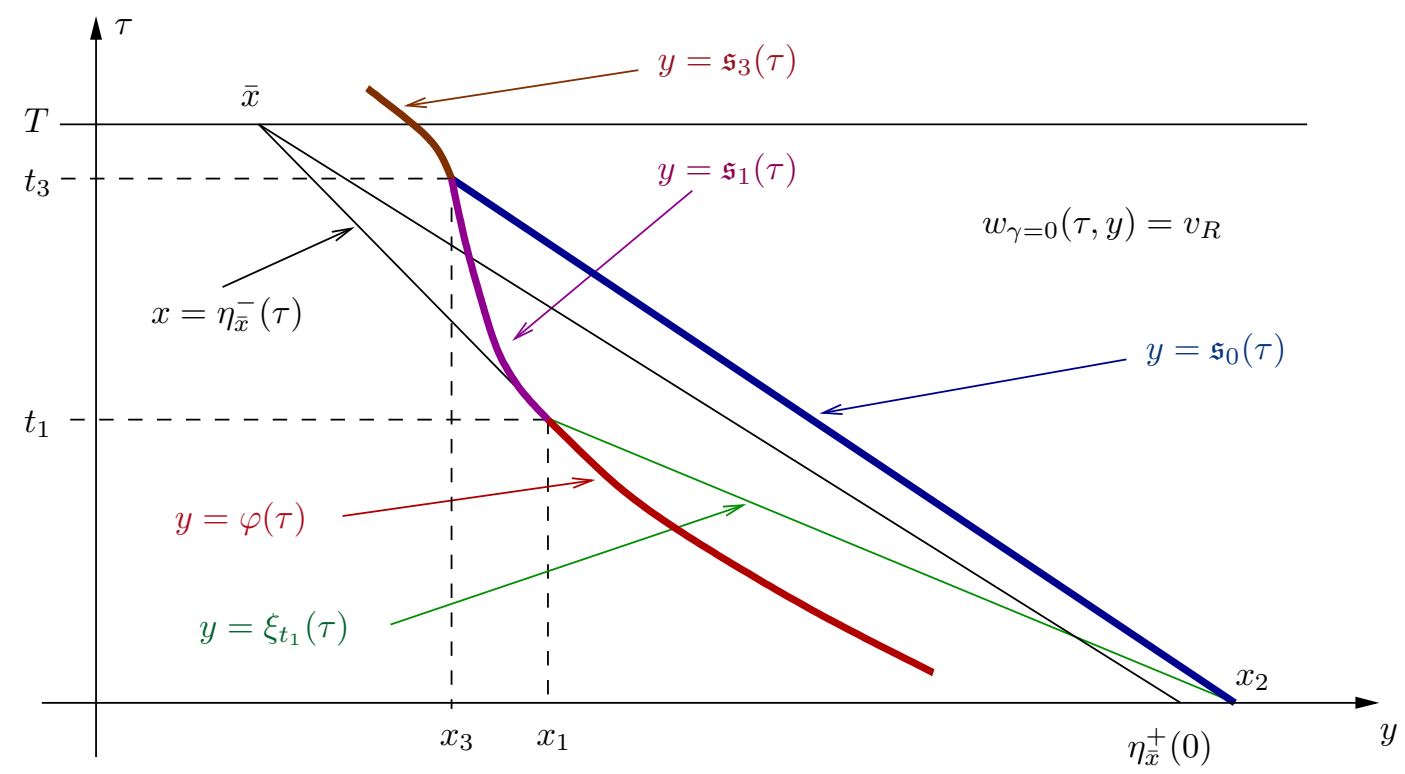

Figure 4: The Remark 3.5

Step 5: Under assumption (3.4), in $\boldsymbol{w}_{\boldsymbol{\gamma}=\mathbf{0}}$ there holds $\overline{\boldsymbol{t}}<\boldsymbol{T}$. Now we use conservation in the region $\Omega_{5}$, defined at (3.22), in order to determine $\bar{t}$. We have

$$
\iint_{\Omega_{5}} \partial_{t} u+\partial_{x} f(u) d x d t=0
$$


so that, by the divergence theorem

$$
\begin{aligned}
0= & \int_{t_{3}}^{\bar{t}}-\mathfrak{q}\left(t, \mathfrak{s}_{3}(t)\right) \mathfrak{s}_{3}^{\prime}(t)+f\left(\mathfrak{q}\left(t, \mathfrak{s}_{3}(t)\right)\right) d t \\
& +\int_{t_{1}}^{t_{3}}-\mathfrak{q}\left(t, \mathfrak{s}_{1}(t)\right) \mathfrak{s}_{1}^{\prime}(t)+f\left(\mathfrak{q}\left(t, \mathfrak{s}_{1}(t)\right)\right) d t \\
& +\int_{t_{1}}^{\bar{t}} \mathfrak{q}\left(t, \eta_{\bar{x}}^{-}(t)\right)\left(\eta_{\bar{x}}^{-}\right)^{\prime}(t)-f\left(\mathfrak{q}\left(t, \eta_{\bar{x}}^{-}(t)\right)\right) d t .
\end{aligned}
$$

Let us compute the three integrals in (3.26). Regarding (3.26a), since Rankine-Hugoniot conditions hold along $x=\mathfrak{s}_{3}(t)$, we get

$$
-\mathfrak{q}\left(t, \mathfrak{s}_{3}(t)\right) \mathfrak{s}_{3}^{\prime}(t)+f\left(\mathfrak{q}\left(t, \mathfrak{s}_{3}(t)\right)\right)=-v_{R} \mathfrak{s}_{3}^{\prime}(t)+f\left(v_{R}\right) \quad \forall t_{3}<t<\bar{t},
$$

and hence

$$
\begin{aligned}
\int_{t_{3}}^{\bar{t}}-\mathfrak{q}\left(t, \mathfrak{s}_{3}(t)\right) \mathfrak{s}_{3}^{\prime}(t)+f(\mathfrak{q}(t & \left.\left., \mathfrak{s}_{3}(t)\right)\right) d t=-v_{R}\left(\mathfrak{s}_{3}(\bar{t})-x_{3}\right)+f\left(v_{R}\right)\left(\bar{t}-t_{3}\right)= \\
& =-v_{R}\left(\bar{x}-x_{3}\right)+v_{R} f^{\prime}\left(v_{L}\right) T-f\left(v_{R}\right) t_{3}+\left[f\left(v_{R}\right)-v_{R} f^{\prime}\left(v_{L}\right)\right] \bar{t} .
\end{aligned}
$$

Now we compute (3.26b). Since Rankine-Hugoniot conditions hold along $\mathfrak{s}_{1}$, we get

$$
\left.-\mathfrak{q}\left(t, \mathfrak{s}_{1}(t)\right) \mathfrak{s}_{1}^{\prime}(t)+f\left(\mathfrak{q}\left(t, \mathfrak{s}_{1}(t)\right)\right)=-\mathfrak{r}\left(t, \mathfrak{s}_{1}(t)+\right) \mathfrak{s}_{1}^{\prime}(t)+f\left(\mathfrak{r}\left(t, \mathfrak{s}_{1}(t)+\right)\right) \quad \forall t \in\right] t_{1}, t_{3}[.
$$

Using again conservation in the region $\Omega_{4}$, see (3.20) we get

$$
\begin{aligned}
\int_{t_{1}}^{t_{3}} & -\mathfrak{q}\left(t, \mathfrak{s}_{1}(t)\right) \mathfrak{s}_{1}^{\prime}(t)+f\left(\mathfrak{q}\left(t, \mathfrak{s}_{1}(t)\right)\right) d t=\int_{t_{1}}^{t_{3}}-\mathfrak{r}\left(t, \mathfrak{s}_{1}(t)+\right) \mathfrak{s}_{1}^{\prime}(t)+f\left(\mathfrak{r}\left(t, \mathfrak{s}_{1}(t)+\right)\right) d t= \\
& =\int_{\gamma}^{t_{3}}-\mathfrak{r}\left(t, \mathfrak{s}_{0}(t)\right) \mathfrak{s}_{0}^{\prime}(t)+f\left(\mathfrak{r}\left(t, \mathfrak{s}_{0}(t)\right)\right) d t+\int_{\gamma}^{t_{1}} \mathfrak{r}\left(t, \xi_{t_{1}}(t)+\right) \xi_{t_{1}}^{\prime}(t)-f\left(\mathfrak{r}\left(t, \xi_{t_{1}}(t)+\right)\right) d t
\end{aligned}
$$

and being

$$
\begin{aligned}
\int_{\gamma}^{t_{3}}-\mathfrak{r}\left(t, \mathfrak{s}_{0}(t)\right) \mathfrak{s}_{0}^{\prime}(t)+f\left(\mathfrak{r}\left(t, \mathfrak{s}_{0}(t)\right)\right) d t & =\int_{\gamma}^{t_{3}}-v_{R}^{b} \mathfrak{s}_{0}^{\prime}(t)+f\left(v_{R}^{b}\right) d t \\
& =\left[-v_{R}^{b} f^{\prime}\left(v_{R}^{b}\right)+f\left(v_{R}^{b}\right)\right]\left(t_{3}-\gamma\right) \\
\int_{\gamma}^{t_{1}} \mathfrak{r}\left(t, \xi_{t_{1}}^{\prime}(t)+\right) \xi_{t_{1}}^{\prime}(t)-f\left(\mathfrak{r}\left(t, \xi_{t_{1}}^{\prime}(t)+\right)\right) d t & =\int_{\gamma}^{t_{1}} v_{L}^{\sharp} \xi_{t_{1}}^{\prime}(t)-f\left(v_{L}^{\sharp}\right) d t \\
& =\left[v_{L}^{\sharp} f^{\prime}\left(v_{L}^{\sharp}\right)-f\left(v_{L}^{\sharp}\right)\right]\left(t_{1}-\gamma\right),
\end{aligned}
$$

and using Rankine-Hugoniot conditions along $\mathfrak{s}_{0}$, we obtain

$$
\begin{aligned}
\int_{t_{1}}^{t_{3}}-\mathfrak{q}\left(t, \mathfrak{s}_{1}(t)\right) \mathfrak{s}_{1}^{\prime}(t)+f\left(\mathfrak{q}\left(t, \mathfrak{s}_{1}(t)\right)\right) d t= & \\
= & {\left[-v_{R} f^{\prime}\left(v_{R}^{b}\right)+f\left(v_{R}\right)\right]\left(t_{3}-\gamma\right)+\left[v_{L}^{\sharp} f^{\prime}\left(v_{L}^{\sharp}\right)-f\left(v_{L}^{\sharp}\right)\right]\left(t_{1}-\gamma\right) . }
\end{aligned}
$$


Regarding (3.26c), observe that $\mathfrak{q}\left(t, \eta_{\bar{x}}^{-}(t)\right)=v_{L}$, so that we obtain

$$
\begin{aligned}
\int_{t_{1}}^{\bar{t}} \mathfrak{q}\left(t, \eta_{\bar{x}}^{-}(t)\right)\left(\eta_{\bar{x}}^{-}\right)^{\prime}(t)-f\left(\mathfrak{q}\left(t, \eta_{\bar{x}}^{-}(t)\right)\right) d t & =\int_{t_{1}}^{\bar{t}} v_{L}\left(\eta_{\bar{x}}^{-}\right)^{\prime}(t)-f\left(v_{L}\right) d t \\
& =\left[v_{L} f^{\prime}\left(v_{L}\right)-f\left(v_{L}\right)\right]\left(\bar{t}-t_{1}\right) .
\end{aligned}
$$

From (3.26), and observing that by construction of $x_{1}, \xi_{t_{1}}(\gamma)$ and $x_{3}$

$$
x_{3}-\bar{x}=\gamma f^{\prime}\left(v_{L}^{\sharp}\right)-f^{\prime}\left(v_{L}\right) T+f^{\prime}\left(v_{R}^{b}\right) t_{3}+\left[f^{\prime}\left(v_{L}\right)-f^{\prime}\left(v_{L}^{\sharp}\right)\right] t_{1},
$$

we obtain

$$
\begin{aligned}
& {\left[f\left(v_{R}\right)-f\left(v_{L}\right)-\left(v_{R}-v_{L}\right) f^{\prime}\left(v_{L}\right)\right] \bar{t}+} \\
& +\left[f\left(v_{L}\right)-f\left(v_{L}^{\sharp}\right)+v_{R}\left(f^{\prime}\left(v_{L}\right)-f^{\prime}\left(v_{L}^{\sharp}\right)\right)+v_{L}^{\sharp} f^{\prime}\left(v_{L}^{\sharp}\right)-v_{L} f^{\prime}\left(v_{L}\right)\right] t_{1}+ \\
& \quad+\left[v_{R} f^{\prime}\left(v_{L}^{\sharp}\right)+v_{R} f^{\prime}\left(v_{R}^{b}\right)-v_{L}^{\sharp} f^{\prime}\left(v_{L}^{\sharp}\right)+f\left(v_{L}^{\sharp}\right)-f\left(v_{R}\right)\right] \gamma=0 .
\end{aligned}
$$

Since

$$
f\left(v_{R}\right)-f\left(v_{L}\right)-\left(v_{R}-v_{L}\right) f^{\prime}\left(v_{L}\right) \neq 0,
$$

due to the fact that $\left(v_{L}^{\sharp}\right)^{\sharp}<v_{R}<v_{L}$, and being

$$
f\left(v_{L}\right)-f\left(v_{L}^{\sharp}\right)=f^{\prime}\left(v_{L}\right)\left(v_{L}-v_{L}^{\sharp}\right),
$$

we recover

$$
\begin{aligned}
\bar{t}=-\frac{\left(v_{R}-v_{L}^{\sharp}\right)\left[f^{\prime}\left(v_{L}\right)-f^{\prime}\left(v_{L}^{\sharp}\right)\right]}{f\left(v_{R}\right)-f\left(v_{L}\right)-\left(v_{R}-v_{L}\right) f^{\prime}\left(v_{L}\right)} t_{1}+ \\
\quad-\frac{v_{R} f^{\prime}\left(v_{L}^{\sharp}\right)+v_{R} f^{\prime}\left(v_{R}^{b}\right)-v_{L}^{\sharp} f^{\prime}\left(v_{L}^{\sharp}\right)+f\left(v_{L}^{\sharp}\right)-f\left(v_{R}\right)}{f\left(v_{R}\right)-f\left(v_{L}\right)-\left(v_{R}-v_{L}\right) f^{\prime}\left(v_{L}\right)} \gamma .
\end{aligned}
$$

Using the expression of $t_{1}$ at (3.5) and due to (3.4), we get $\bar{t} \leq T$ if $\gamma=0$. As $v_{R}<0<v_{R}^{b}<v_{L}^{\sharp}$ the assumption $(\mathbf{F}) \cdot \mathbf{b}$ ensures that the coefficient in front of $\gamma$ is negative. Then it is possible to find $\gamma=\gamma^{*} \in\left[t^{*}, 0\right]$ in order to get $\bar{t}=T$. It follows that $u_{3}=w_{\gamma}^{0}$ with $\gamma=\gamma^{*}$ in (3.17), and $u=w_{\gamma}$ with $\gamma=\gamma^{*}$ in (3.23) are, respectively, the explicit formulation of an initial datum $u_{3}$ and a solution $u$ to (3.11) attaining $v$ at time $T$.

\subsection{Proof of Theorem 2}

Assume that $v$ satisfies to all conditions in the statement of Theorem 2. We consider the partition of $\left[\alpha_{0}, \beta_{0}\right]$ into maximal subintervals in which $v$ is continuous, namely $\left[\alpha_{0}, \beta_{0}\right]=$ $\left.\left.\left.\cup_{n \geq 1} I_{n}\right], I_{n}=\right] x_{n}, x_{n+1}\right]$. Define $J_{n}=\left\{x \in I_{n}: D_{x} f^{\prime}(v(x))>1 / T\right\}$. Consider now the function

$$
v_{n}(x)= \begin{cases}v\left(x_{n}^{+}\right) & \text {if } \alpha_{0} \leq x \leq x_{n} \\ v(x) & \text { if } x \in I_{n} \\ v\left(x_{n+1}^{+}\right) & \text {if } x_{n+1}<x \leq \beta_{0}\end{cases}
$$


and observe that $v_{n}$ satisfies the hypothesis of one of the three Lemmas 3.1, 3.2 or 3.3. Therefore, there exist a control $u_{c}^{n}$ which we can use as initial condition in (1.1) to attain the profile $v_{n}$ at time $T$. Under the hypothesis of Theorem 2, in particular, in absence of nesting structure, we are sure that the control function $u_{c}^{n}$ is constant outside the interval $Y_{n}=\left[\eta_{x_{n}^{+}}(0), \eta_{x_{n+1}^{+}}(0)[\right.$. Therefore, the function

$$
u_{c}(y)=\sum_{n=0}^{N} u_{c}^{n}(y) \mathbb{1}_{Y_{n}}(y),
$$

can be used as initial condition in (1.1) to attain the profile $v$ at time $T$.

\section{Application to Kynch's sedimentation model}

In this section we give an explicit example on the application of Lemma 3.3 to a real life problem. We consider the classical model for sedimentation proposed by Kynch in [17], see also [6] for an introduction to the model from an historical point of view and a short account of the related literature.

The model describes the sedimentation of the solid part of a suspension in a cylindrical batch of height $L$. The unknown function in the equation is the local solid fraction of the suspension, $u$, which varies between 0 and a maximal value $u_{\max }$. For technical reasons (presence of a suitable mixing device) the only relevant space dimension in the problem is the height above the bottom of the batch, $x \in[0, L]$. The Ansatz used by Kynch is that the velocity of sedimentation, $V$, at any level $x$ depends on the value of the local solid fraction $u$, so that the flux function is given by $f(u)=V(u) u$. If we write $t$ for the time variable, the model takes the form of a scalar conservation law in one space dimension

$$
\partial_{t} u+\partial_{x}(V(u) u)=0 .
$$

Given the parametrization of the batch it is natural to impose that $f$ is negative for $u \in\left[0, u_{\max }\right]$ and attains zero for $u=0$ and $u=u_{\max }$. One can find many different constitutive equations for $V$ in the existing literature. In this example, to allow for explicit computations, we take $u_{\max }=1$ and we consider $V(u)=-(1-u)^{2}$, which leads to the flux function $f(u)=-u(1-u)^{2}$, so that (4.1) becomes

$$
\partial_{t} u-\partial_{x}\left(u(1-u)^{2}\right)=0 .
$$

Such flux function suffers from an inflection point at $u=2 / 3$, but it does not satisfy the conditions we fixed in $(\mathbf{F}) \cdot \mathbf{b}$ in order to simplify our presentation. Nevertheless all the results in the previous sections apply to this case. In analogy with the notation introduced in (1.7)(1.9), for any $v$ we have

$$
v^{\sharp}=2(1-v), \quad\left(v^{\sharp}\right)^{\sharp}=2(2 v-1), \quad v^{b}=1-\frac{v}{2} .
$$

Since the volume fraction of solid $u$ varies in the interval $[0,1]$, we need $v \in[1 / 2,3 / 4]$ in order to have $v^{\sharp}$ and $\left(v^{\sharp}\right)^{\sharp}$ in the same interval $[0,1]$. 
Fix $T=2$, and consider the final profile

$$
v_{2}(x)= \begin{cases}1 / 2 & \text { if } \quad x \leq 1 / 2, \\ \frac{4 \sqrt{7}-\sqrt{4+3 \sqrt{15-28 x}}}{6 \sqrt{7}} & \text { if } \quad 1 / 2<x \leq \frac{59}{112}, \\ \frac{224-13 \sqrt{154}}{84} & \text { if } \quad x>\frac{59}{112} .\end{cases}
$$

We illustrate how the backward reconstruction exploited in the proof of Lemma 3.3, allows to construct an initial datum $u_{0}$ such that the solution $u=u(t, x)$ to $(4.2)$ with $u(0, \cdot)=u_{0}$ fulfills $u(2, \cdot)=v_{2}$.

The target profile suffers from a jump discontinuity at $\bar{x}=59 / 112$. In the following we call

$$
v_{L} \doteq v(59 / 112)=\frac{56-\sqrt{154}}{84}, \quad v_{R} \doteq v(59 / 112+)=\frac{224-13 \sqrt{154}}{336} .
$$

A straightforward computation, see (4.3), gives us

$$
v_{L}^{\sharp}=\frac{28+\sqrt{154}}{42}, \quad\left(v_{L}^{\sharp}\right)^{\sharp}=\frac{14-\sqrt{154}}{21},
$$

so we can check that $v_{R} \in\left[\left(v_{L}^{\sharp}\right)^{\sharp}, v_{L}\right]$. For future use we also compute

$$
v_{R}^{b}=\frac{448+13 \sqrt{154}}{672} .
$$

Observe that, being $D_{x} f^{\prime}(v(x))=(2 \sqrt{15-28 x})^{-1}$, the interval $J$ at (3.1) is non empty, $\sup J=$ $\bar{x}$ and $\inf J=\hat{x}=1 / 2$, and $t_{1}=1$, see (3.5). The convex envelope of the lines $\eta_{x}$ defined at (1.14c) for $x \in J=] 1 / 2,59 / 112]$ is

$$
\varphi(t)=\frac{1}{112} t^{2}+\frac{1}{4} t, \quad t \leq 1 \doteq t_{1},
$$

Moreover, being $\left(v_{L}^{\sharp}\right)^{\sharp}<v_{R}<v_{L}$ and

$$
-\frac{\left(v_{R}-v_{L}^{\sharp}\right)\left[f^{\prime}\left(v_{L}\right)-f^{\prime}\left(v_{L}^{\sharp}\right)\right]}{f\left(v_{R}\right)-f\left(v_{L}\right)-\left(v_{R}-v_{L}\right) f^{\prime}\left(v_{L}\right)} t_{1}=\frac{16}{9}<2=T,
$$

the assumption (3.4) is satisfied. Hence, all hypotheses of Lemma 3.3 are fulfilled. Plugging $\bar{t}=T=2$ and $t_{1}=1$ in equation (3.28), we easily find the value $\gamma=\gamma^{*}$ which allows us to construct the initial condition

$$
\begin{gathered}
\gamma^{*}=-\left[\frac{f\left(v_{R}\right)-f\left(v_{L}\right)-\left(v_{R}-v_{L}\right) f^{\prime}\left(v_{L}\right)}{v_{R} f^{\prime}\left(v_{L}^{\sharp}\right)+v_{R} f^{\prime}\left(v_{R}^{b}\right)-v_{L}^{\sharp} f^{\prime}\left(v_{L}^{\sharp}\right)+f\left(v_{L}^{\sharp}\right)-f\left(v_{R}\right)}\right] . \\
\cdot\left[2+\frac{\left(v_{R}-v_{L}^{\sharp}\right)\left[f^{\prime}\left(v_{L}\right)-f^{\prime}\left(v_{L}^{\sharp}\right)\right]}{f\left(v_{R}\right)-f\left(v_{L}\right)-\left(v_{R}-v_{L}\right) f^{\prime}\left(v_{L}\right)}\right]= \\
=-\frac{6823432+5667200 \sqrt{154}}{391268487} \approx-0.197183 .
\end{gathered}
$$


From equations (3.13) and (3.14) we get

$$
x_{1}=\frac{29}{112}, \quad x_{2}=\frac{3}{16} .
$$

With the choice (4.5) of $\gamma=\gamma^{*}$ and using (3.16), we get

$$
\begin{aligned}
\mathfrak{a} & =x_{1}+f^{\prime}\left(v_{L}^{\sharp}\right)\left(\gamma^{*}-1\right)=\frac{1166007253-6476800 \sqrt{154}}{6260295792} \approx 0.173415 \\
\mathfrak{s}_{0}(0) & =\mathfrak{a}-f^{\prime}\left(v_{R}^{b}\right) \gamma^{*}=\frac{4734091037+32282800 \sqrt{154}}{25041183168} \approx 0.205051 .
\end{aligned}
$$

Hence, from (3.17), we get that an initial datum by means of which we get $v_{2}$ at time $T=2$ is given by

$$
u_{0}(x)= \begin{cases}1 / 2 & \text { if } \quad x \leq 0, \\ \frac{2 \sqrt{7}+\sqrt{4+3 \sqrt{1-4 x}}}{3 \sqrt{7}} & \text { if } \quad 0<x \leq 3 / 16, \\ \frac{2}{3}+\sqrt{\frac{\gamma^{*}+3 x-3 \mathfrak{a}}{\gamma^{*}}} & \text { if } \quad 3 / 16<x \leq \mathfrak{s}_{0}(0), \\ \frac{224-13 \sqrt{154}}{336} & \text { if } \quad x>\mathfrak{s}_{0}(0) .\end{cases}
$$

\section{More complex structures}

This section consists of two parts. First, in Section 5.1 we present a simple case of nesting in a very smooth framework. We state some conditions for attainability and we provide an explicit example. This presentation is of course far from being exhaustive but it is sufficient to illustrate the main features of the problem.

In section 5.2 we present some ideas toward the construction of a recursive procedure which could be used to simplify the problem in more general situations. The recursive procedure naturally leads to state an open problem in the general theory of scalar conservation laws.

\subsection{Two nested contact discontinuities}

Let $v$ be an attainable profile suffering from a jump discontinuity at $x=\bar{x}$, connecting the states $v_{L}=v\left(\bar{x}^{-}\right)$, and $v_{R}=v\left(\bar{x}^{+}\right)=v_{L}^{\sharp}$. We assume that tracing back the candidate backward characteristics $\eta_{x}$, one finds that there exist $x_{1}$ and $x_{2}$ such that

- for all $\left.\left.x \in J_{\bar{x}}^{L}=\right] x_{1}, \bar{x}\right]$ the lines $\eta_{x}$, graze from the left contact discontinuity $\tau \mapsto \varphi_{1}(\tau)$;

- for all $\left.\left.x \in J_{\bar{x}}^{R}=\right] \bar{x}, x_{2}\right]$ the lines $\eta_{x}$, graze from the left contact discontinuity $\tau \mapsto \varphi_{2}(\tau)$.

It is well known that once a left contact discontinuity appears in the solution it persists until it interact with second left contact or with a shock. This means that the admissible profile $v$ must suffer from a second jump discontinuity, taking place at $x=x_{2}$. The Proposition below, whose proof is straightforward in the light of the analysis in the preceding Sections, formalizes these necessary conditions. 
Proposition 5.1. In the hypothesis above there exists $\overline{\bar{x}} \in \mathbb{R}$, such that

- $\overline{\bar{x}}>\bar{x}$ and $v\left(\overline{\bar{x}}^{-}\right) \neq v\left(\overline{\bar{x}}^{+}\right)$;

- $\left(v\left(\overline{\bar{x}}^{-}\right), v\left(\overline{\bar{x}}^{+}\right)\right)$is an admissible jump in the sense of condition 4 of Theorem 1.

- The set $J_{\overline{\bar{x}}} \doteq\left\{\bar{x}<x \leq \overline{\bar{x}}: D_{x} f^{\prime}(v(x))>1 / T\right\}$, analogous to the set $J$ defined in (3.1), is exactly $] \bar{x}, \overline{\bar{x}}]$ and for all $x \in J_{\overline{\bar{x}}}$ there holds $D^{+}\left(D_{x} f^{\prime}(v(x))\right)>0$.

Regarding the lines $\tau \mapsto \xi_{t(x)}(\tau)$ defined as in (2.8) as the candidate backward characteristics from $\left(t(x), \varphi_{1}(t(x))\right)$, two configurations are possible. If they do not cross each other in positive time, it means that the left contact discontinuities $\varphi_{1}$ and $\varphi_{2}$ are essentially disconnected one from the other. Otherwise, we conclude that the lines $\xi_{t(x)}$ graze from $\varphi_{2}$ and nesting occurs (see figure 5). This second case is the one we are interested in. Without surprise, we have that

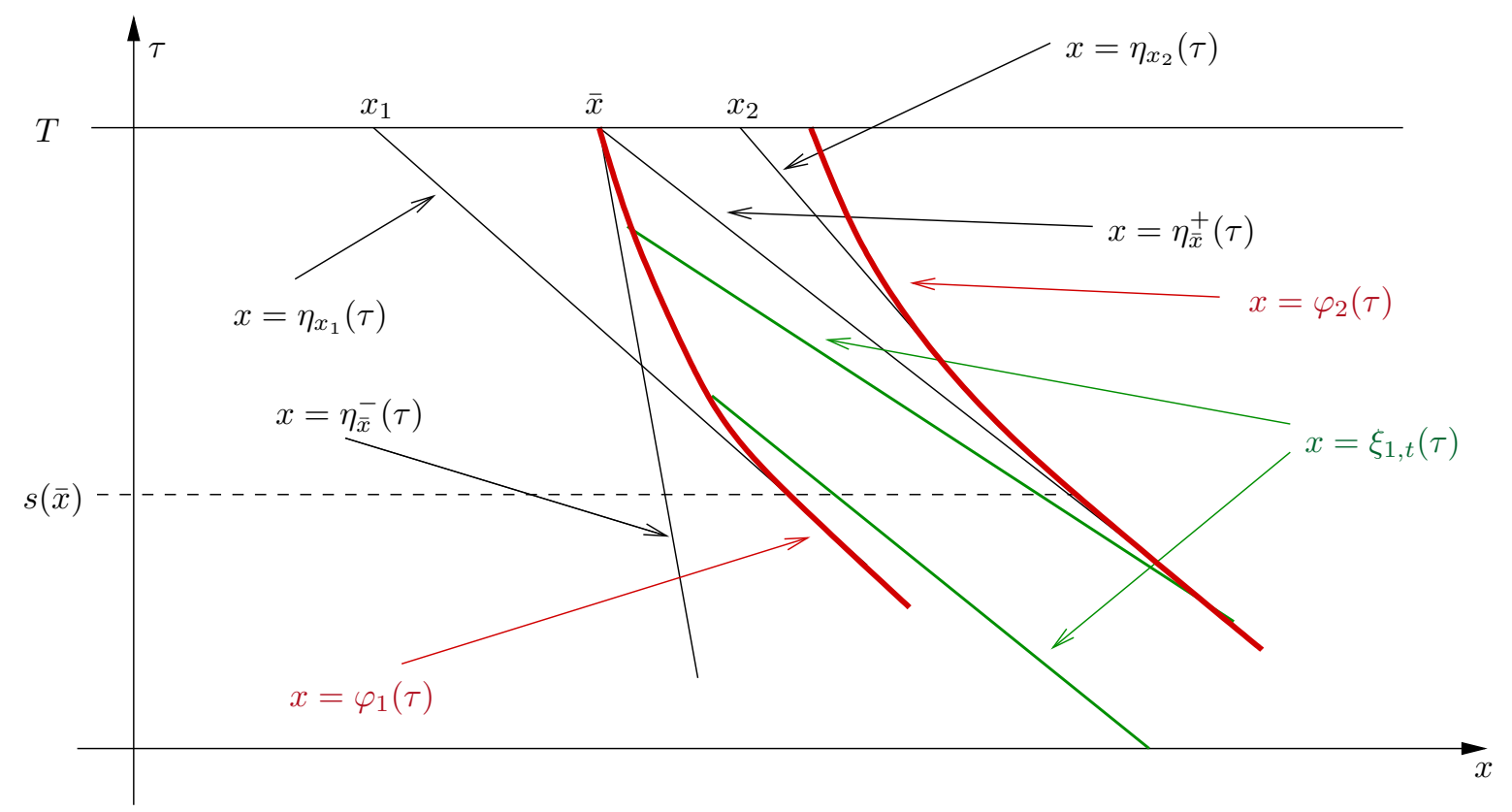

Figure 5: Configuration with two consecutive contact discontinuities

in a configuration like the one at figure 5 not only the jump in $\bar{x}$ must be admissible in the sense of Theorem 1, but there is also a compatibility condition among the values of $v$ in a left and in a right neighbourhood of $\bar{x}$.

Theorem 3. Let $v$ be a piecewise $\mathcal{C}^{2}$ attainable profile at time $T$, suffering from a jump at $x=\bar{x}$ with left and right states $v_{L}$ and $v_{R}=v_{L}^{\sharp}$, respectively. Assume that in a neighbourhood of $(T, \bar{x})$ the configuration described above and illustrated in figure 5 occurs. Then

$$
\lim _{x \rightarrow \bar{x}^{+}} D_{x} f^{\prime}\left(v(x)=\lim _{x \rightarrow \bar{x}^{-}} \frac{\left[D_{x} f^{\prime}(v(x))\right]^{2} D_{x} f^{\prime}\left(v^{\sharp}(x)\right)}{D_{x x}^{2} f^{\prime}(v(x))\left[f^{\prime}\left(v_{L}\right)-f^{\prime}\left(v_{R}\right)\right]} .\right.
$$


Proof. Let $s(\bar{x}) \in] 0, T\left[\right.$ be such that $\eta_{\bar{x}}^{+}(s(\bar{x}))=\varphi_{2}(s(\bar{x}))$. Due to (2.6) it turns out that

$$
s(\bar{x})=T-\lim _{x \rightarrow \bar{x}^{+}} \frac{1}{D_{x} f^{\prime}(v(x))} .
$$

Now we compute $s(\bar{x})$ using the fact that there exists $t_{0}<s(\bar{x})$ such that for $\left.\left.\tau \in\right] t_{0}, s(\bar{x})\right]$ the curve $x=\varphi_{2}(\tau)$ is the convex envelope of the maximal characteristics grazing from $x=\varphi_{1}(\tau)$. Let $I$ be a suitable left neighbourhood of $\bar{x}$ such that $x=\varphi_{1}(\tau)$ is the tangent envelope of the backward characteristics grazing from $(T, x), x \in I$, so that (2.7) holds with $\varphi=\varphi_{1}$. Hence, we can write the maximal backward characteristics from $\varphi_{1}$ as

$$
\left.\xi_{t(x)}(\tau)=\varphi_{1}(t(x))+f^{\prime}\left(v^{\sharp}(x)\right)\right)(\tau-t(x)) .
$$

Such a line intersect $\eta_{\bar{x}}^{+}$at time

$$
s(\bar{x}, x)=-\frac{\varphi_{1}(t(x))-\bar{x}-\left(f^{\prime}\left(v^{\sharp}(x)\right) t(x)-f^{\prime}\left(v_{R}\right) T\right)}{f^{\prime}\left(v^{\sharp}(x)\right)-f^{\prime}\left(v_{R}\right)} .
$$

Letting $x \rightarrow \bar{x}^{-}$we get

$$
s(\bar{x})=T+\lim _{x \rightarrow \bar{x}^{-}} \frac{D_{x x}^{2} f^{\prime}(v(x))\left[\left(f^{\prime}\left(v_{R}\right)-f^{\prime}\left(v_{L}\right)\right]\right.}{\left[D_{x} f^{\prime}(v(x))\right]^{2} D_{x} f^{\prime}\left(v^{\sharp}(x)\right)},
$$

that together with (5.2) gives (5.1)

In the spirit of Proposition 2.3 and having in mind the configuration at figure 5, one can easily formulate conditions on the values of $v$ on $J_{\bar{x}}^{L}$ in order that the tangent envelope of the the family of lines $\left\{\xi_{t(x)}\right\}_{x \in J_{\bar{x}}^{L}}$ at (5.3), turns out to be a convex, Lipschitz continuous curve. This is the object of the remaining part of this Section. Please notice that the analysis is only meaningful in an extremely smooth setting.

We add here, for the reader's convenience, the expression of the derivative of the function $x \mapsto \xi_{t(x)}(0)$ at $(2.9)$. We write $\xi_{x}$ for $\xi_{t(x)}$ to simplify the notation.

$$
\frac{d \xi_{x}}{d x}(0)=-D_{x} f^{\prime}\left(v^{\sharp}(x)\right) t(x)-\frac{D_{x x}^{2} f^{\prime}(v(x))}{\left[D_{x} f^{\prime}(v(x))\right]^{2}}\left(f^{\prime}\left(v^{\sharp}(x)\right)-f^{\prime}(v(x))\right)
$$

From the basic hypothesis in Proposition 2.4 we deduce that the first term in the expression above is positive, while the second is negative. This justify the following definitions

- Let $\tilde{x}$ be defined as in (2.10). $J=[\tilde{x}, \bar{x}]$ contains $J_{\bar{x}}^{L}$. We call $\underline{x}$ the first point of local maximum of $x \mapsto \xi_{x}(0)$ in $J_{\bar{x}}^{L}$

$$
\underline{x}=\min \left\{x \in J: \frac{d \xi_{x}}{d x}(0)=0, \frac{d^{2} \xi_{x}}{d x^{2}}(0)<0\right\} .
$$

This point exists by Rolle's theorem and the fact that the function $x \mapsto \xi_{x}(0)$ cannot take values smaller that $\eta_{\bar{x}}^{+}(0)$ in the interval $J=[\tilde{x}, \bar{x}]$ otherwise given $y$ in the interior of $J$ 
the line $\xi_{y}$ would cross $\xi_{\tilde{x}}$ on the left of $\eta_{\bar{x}}^{+}$and this contradicts the necessary condition for attainability stated in Proposition 2.2. This argument actually proves that $\underline{x}$ is the only point of maximum of $x \mapsto \xi_{x}(0)$ in the interval $J$. Assume by contradiction that there exists a second local maximum at $y, y>\underline{x}$. Then if, for example, $\xi_{y}(0)>\xi_{\underline{x}}(0)$ we have that some of the lines $\xi_{x}$, originated at time $t(x)<t(y)$, cross on the left of $\xi_{y}$ and this is not admissible because of Proposition 2.2. If $\xi_{y}(0)<\xi_{\underline{x}}(0)$ the situation is similar.

- If some of the lines $\xi_{x}$ cross $\eta_{\bar{x}}^{+}$at positive time then the function $x \mapsto \xi_{x}(0)$ has to be decreasing in a left neighbourhood of $\bar{x}$. Then we call $(\bar{x}, v) \mapsto \bar{t}(\bar{x}, v)$ the function which associates to a final profile $v$ and one of its points of jumps $\bar{x}$ the latest time at which the backward characteristic lines $\xi_{x}$ may cross $\eta_{\bar{x}}^{+}$

$$
\bar{t}(\bar{x}, v)=T+\lim _{x \rightarrow \bar{x}^{-}} \frac{D_{x x}^{2} f^{\prime}(v(x))}{\left[D_{x} f^{\prime}(v(x)]^{2} D_{x} f^{\prime}\left(v^{\sharp}(x)\right)\right.}\left(f^{\prime}\left(v^{\sharp}(x)\right)-f^{\prime}(v(x))\right) .
$$

Proposition 5.2. Assume that $T>\bar{t}(\bar{x}, v)$, that all the hypothesis of Proposition 2.4 are verified and that

$$
\frac{d^{2} \xi_{x}}{d x^{2}}(0)-\frac{D_{x x}^{2} f^{\prime}\left(v^{\sharp}(x)\right)}{D_{x} f^{\prime}\left(v^{\sharp}(x)\right)} \frac{d \xi_{x}}{d x}(0)<0, \quad \text { for } x \in[\underline{x}, \bar{x}],
$$

with $\underline{x}$ defined in (5.5) here above. Then the tangent envelope of the lines

$$
\xi_{x}(t)=\varphi_{1}(t(x))+f^{\prime}\left(v^{\sharp}(x)\right)(t-t(x)), \quad x \in[\underline{x}, \bar{x}], \quad 0 \leq t \leq t(\underline{x}),
$$

is a strictly convex $C^{1}$ curve $x=\varphi_{2}(s)$, defined in a suitable interval $\left[s_{0}, s_{1}\right] \subseteq[0, T]$.

The proof is omitted, as it follows exactly the same lines as the proof of Proposition 2.4.

\subsubsection{An example}

Consider again the Kynch model for sedimentation (4.2), and let

$$
v_{3}(x)= \begin{cases}1 / 2 & \text { if } \quad x \leq \frac{3}{4}, \\ \frac{1}{6}\left[4-\sqrt{\frac{5}{14}+\frac{3}{14} \sqrt{93-112 x}}\right] & \text { if } \quad \frac{3}{4}<x \leq \frac{93}{112}, \\ \frac{14+\sqrt{10+3 \sqrt{169-196 x}}}{21} & \text { if } \frac{93}{112}<x \leq \frac{165}{196}, \\ \frac{2}{7} & \text { if } \quad x>\frac{165}{196},\end{cases}
$$

be a candidate final profile at time $T=3$ for a solution to (4.2). In order to reconstruct an initial datum for (4.2) from $v_{3}$, we trace backwardly the lines $\left\{\eta_{x}\right\}_{x \in] 3 / 4,165 / 196]}$ (see figure 5 , where $\bar{x}=93 / 112$ )

$$
\eta_{x}(t)=x+f^{\prime}\left(v_{3}(x)\right)(t-3)= \begin{cases}x+\frac{17-\sqrt{93-112 x}}{56}(t-3) & \text { if } \quad 3 / 4<x \leq \frac{93}{112}, \\ x+\frac{13-\sqrt{169-196 x}}{49}(t-3) & \text { if } \quad \frac{93}{112}<x \leq \frac{165}{196}\end{cases}
$$


The tangent envelope of $\eta_{x}$ for $\left.\left.x \in\right] 3 / 4,93 / 11\right]$ can be easily computed by means of (2.6)-(2.7), and it turns out to be the convex curve

$$
\varphi_{1}(t)=\frac{1}{112} t^{2}+\frac{1}{4} t, \quad 0 \leq t \leq 3 .
$$

Such a curve is a candidate left contact discontinuity in the (candidate) solution $u=u(t, x)$ to (4.2) attaining $v_{3}$ at time $T=3$. The left and right states of $x=\varphi_{1}(t)$, say $u_{1}(t)$ and $u_{1}^{\sharp}(t)$, respectively, must fulfil

$$
f^{\prime}\left(u_{1}(t)\right)=\varphi_{1}^{\prime}(t)=\frac{f\left(u_{1}^{\sharp}(t)\right)-f\left(u_{1}(t)\right)}{u_{1}^{\sharp}(t)-u_{1}(t)},
$$

and hence we obtain that

$$
u_{1}(t)=\frac{1}{6}\left(4-\sqrt{\frac{14-3 t}{14}}\right), \quad u_{1}^{\sharp}(t)=\frac{1}{3}\left(2+\sqrt{\frac{14-3 t}{14}}\right)
$$

hold. Now, trace backwardly the lines $\left\{\xi_{1, t}\right\}_{t \in[0,3]}$ (see figure 5),

$$
\xi_{1, t}(\tau) \doteq \varphi_{1}(t)+f^{\prime}\left(u_{1}^{\sharp}(t)\right)(\tau-t)=-\frac{1}{16} t^{2}+\frac{1}{4} t+\frac{1}{14} t \tau,
$$

that are the candidate backward maximal characteristics from $\left(t, \varphi_{1}(t)\right)$ in the candidate solution $u=u(t, x)$ that we would like to reconstruct. It turns out that

$$
\xi_{1,3}(7 / 4)=\lim _{x \rightarrow 93 / 112+} \eta_{x}(7 / 4),
$$

where $7 / 4=s(\bar{x})$ in figure 5 . Hence we can consider the tangent envelope of the family of lines

$$
\left.\left.\left\{\eta_{x}: x \in\right] 93 / 112,165 / 196\right]\right\} \cup\left\{\xi_{1, t}: t \in[2,3]\right\} .
$$

With an easy computation we get the convex curve

$$
\varphi_{2}(t)=\left(\frac{1}{7} t+\frac{1}{2}\right)^{2}, \quad t \in[0,2],
$$

which is another candidate left contact discontinuity in the solution $u$ (see figure 5 again). The (candidate) forward left characteristics grazing from $\left(t, \varphi_{2}(t)\right)$ for $t \in[0,7 / 4]$ are exactly the (candidate) backward maximal characteristics from $\left(t, \varphi_{1}(t)\right)$ for $t \in[2,3]$ (nesting configuration). Now, observe that

$$
\frac{f\left(v_{3}(165 / 196+)\right)-f\left(v_{3}(165 / 196)\right)}{v_{3}(165 / 196+)-v_{3}(165 / 196)}=\frac{11}{49}=\varphi_{2}^{\prime}(2) .
$$

Hence, we can redefine $\varphi_{2}(\cdot)$ as

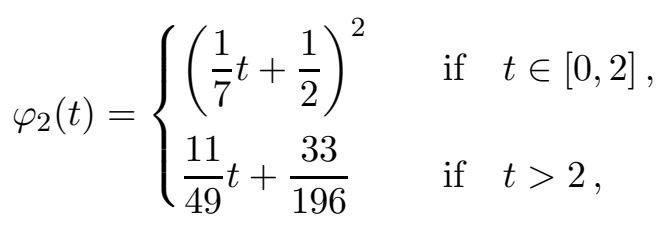


and it turns out to be a (candidate) left contact discontinuity with left and right states

$$
\begin{aligned}
& u_{2}(t)= \begin{cases}\frac{14+2 \sqrt{2(14-3 t)}}{21} & \text { if } \quad 0 \leq t<2, \\
\frac{6}{7} & \text { if } t \geq 2,\end{cases} \\
& u_{2}^{\sharp}(t)= \begin{cases}\frac{14-4 \sqrt{2(14-3 t)}}{21} & \text { if } \quad 0 \leq t<2, \\
\frac{2}{7} & \text { if } t \geq 2,\end{cases}
\end{aligned}
$$

respectively. The lines $\left\{\xi_{1, t}\right\}_{t \in[0,2[}$ do not intersect each other in the time interval $[0,2[$, and the same happens for the family of lines

$$
\xi_{2, t}(\tau)=\varphi_{2}(t)+f^{\prime}\left(u_{2}^{\sharp}(t)\right)(\tau-t)= \begin{cases}\left(\frac{1}{7} t+\frac{1}{2}\right)^{2}+\frac{8 t-21}{49}(\tau-t) & \text { if } t \in[0,2[, \\ \frac{33}{196}+\frac{16}{49} t-\frac{5}{49} \tau & \text { if } t \geq 2,\end{cases}
$$

that are the candidate backward maximal characteristics from $\left(t, \varphi_{2}(t), t \in[0,3]\right.$. It follows that we can reconstruct the initial datum $u_{0}$ which is driven to $v_{3}$ by means of (4.2) by using the method of characteristics, and thus obtain

$$
u_{0}(x)=\left\{\begin{array}{lll}
1 / 2 & \text { if } \quad x \leq 0, \\
\frac{2 \sqrt{7}+\sqrt{4+3 \sqrt{1-4 x}}}{3 \sqrt{7}} & \text { if } \quad 0<x \leq 1 / 4, \\
\frac{14-2 \sqrt{16+3 \sqrt{23-28 x}}}{21} & \text { if } \quad 1 / 4<x \leq 23 / 28, \\
2 / 7 & \text { if } \quad x>23 / 28 .
\end{array}\right.
$$

\subsection{A recursive procedure}

In this section we reformulate the problem of the backward reconstruction of a nesting of contact discontinuities in terms of a recursive procedure. This different point of view leads to a very natural necessary condition for attainability and makes clear the relation between the description of attainable states and an open problem for general scalar conservation laws.

The main idea in the recursive procedure is the following. Let $v$ be a candidate admissible profile suffering from a jump discontinuity at $x=\bar{x}$ between the states $v_{L}$ and $v_{R}$. Moreover, assume that backward characteristic from a left neighbourhood $J$ of $\bar{x}$ do intersect, and that their tangent envelope is a convex curve $x=\varphi_{1}(\tau)$, defined in a left neighbourhood of $t_{1} \leq T$, with $t_{1}$ given by (3.5). Call $u_{1, R}(t)$ the state at the right of $\varphi_{1}$ at time $t$ and let $\xi_{1, t}$ be the candidates backward characteristics from $\left(t, \varphi_{1}(t)\right)$, defined as $\xi_{t}$ in $(2.5)$ with $\varphi=\varphi_{1}$ and $u_{R}(t)=u_{1, R}(t)$. This implies that using the parametrization $(2.8)$ we have $u_{1, R}(t(x))=v^{\sharp}(x)$.

We are interested in the case in which the lines $\xi_{1, t}$ admit a convex tangent envelope $\tau \mapsto \varphi_{2}(\tau)$, along which any possible backward reconstruction of $v$ experiences a contact 
discontinuity. However, in this different approach we do not try to construct $\varphi_{2}$ directly. The idea is to construct on a left closed neighbourhood of $\varphi_{1}\left(t_{1}\right), J_{1}$, a fictious profile $w_{1}$ at $t=t_{1}$ in which the discontinuity at $\varphi_{1}\left(t_{1}\right)$ does not appear, and then try to iterate the method. We proceed in the following way (see figure 6).

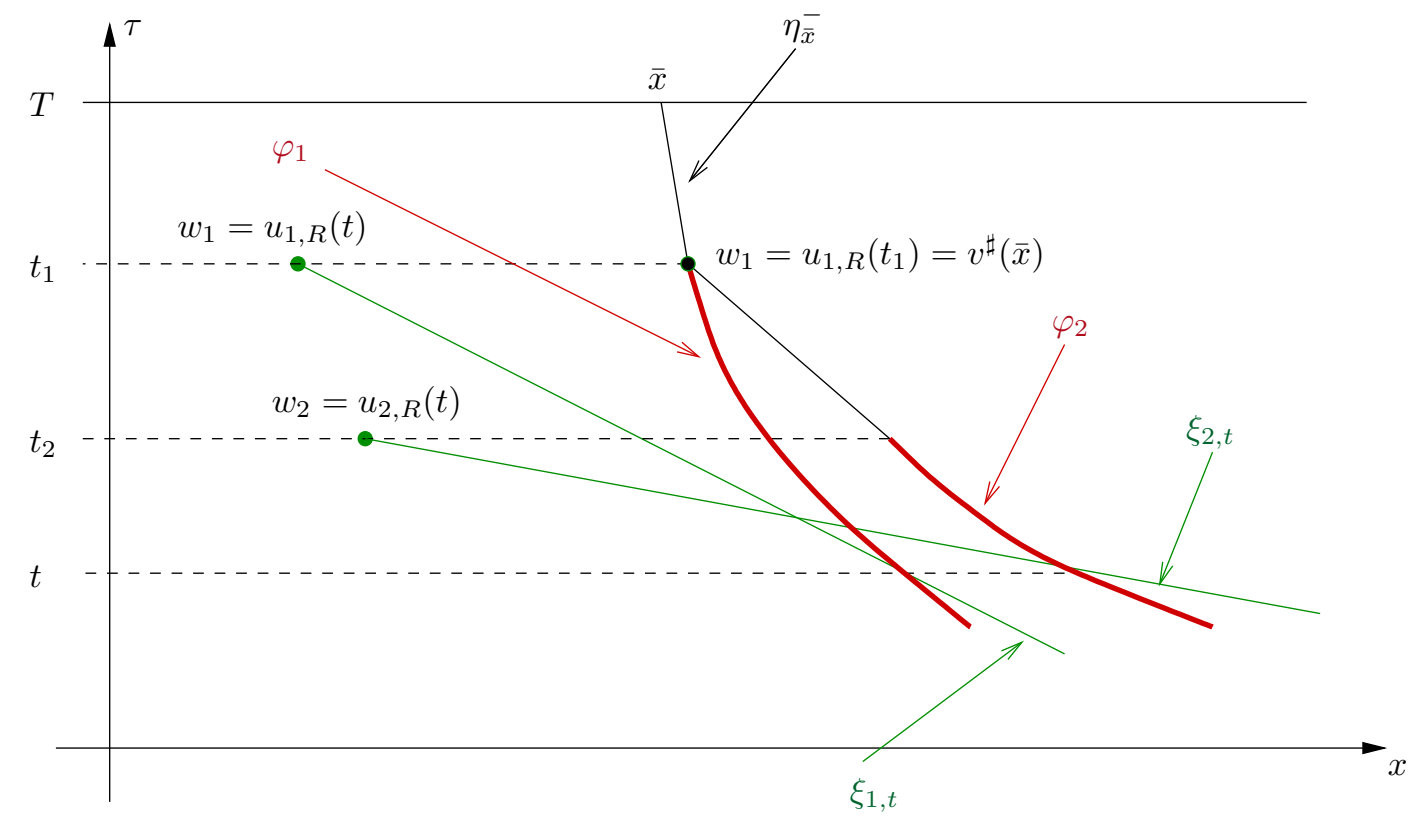

Figure 6: The recursive procedure at Subsection 5.2

1. First step. We define

$$
w_{1}\left(\xi_{1, t(x)}\left(t_{1}\right)\right)=v^{\sharp}(x),
$$

so that

$$
w_{1}\left(\xi_{1, t}\left(t_{1}\right)\right)=u_{1, R}(t) .
$$

In such a way, considering $w_{1}$ as a part of a final profile at time $t=t_{1}$, the candidate maximal backward characteristics from $\left(t_{1}, x\right), x \in J_{1}$, do coincide with $\xi_{1, t}$, i.e., if $x=$ $\xi_{1, t}\left(t_{1}\right)$, then

$$
x+f^{\prime}\left(w_{1}(x)\right)\left(\tau-t_{1}\right)=\xi_{1, t}(\tau) \quad \forall \tau .
$$

2. Second step. By construction, the tangent envelope of the maximal backward characteristics from $\left(t_{1}, x\right), x \in J_{1}$, is exactly $x=\varphi_{2}(\tau)$, and hence $t_{2}$ can be expressed in terms of $w_{1}$ as

$$
t_{2}=t_{1}-\frac{1}{\left.D_{x}^{-} f^{\prime}\left(w_{1}(x)\right)\right|_{x=\varphi_{1}\left(t_{1}\right)}} .
$$

At time $t_{2}$ we are now able to construct a candidate final profile $w_{2}$ defined in a left neighbourhood $J_{2}$ of $\varphi_{2}\left(t_{2}\right)$ using the same method exploited to construct $w_{1}$, and hence considering the lines

$$
\xi_{2, t}(\tau)=\varphi_{2}(t)+f^{\prime}\left(u_{2, R}(t)\right)(\tau-t),
$$


where $u_{2, R}(t)$ is the state at the right of $\varphi_{2}$ choose so that, if $\varphi_{2}^{\prime}(t)=f^{\prime}\left(u_{2, L}(t)\right)$, then $u_{2, R}(t)=\left(u_{2, L}(t)\right)^{\sharp}$. Then $w_{2}$ is defined as

$$
w_{2}\left(\xi_{2, t}\left(t_{2}\right)\right)=u_{2, R}(t) .
$$

3. Now it is clear how we can proceed. Once we reconstruct the convex curve $x=\varphi_{n}(\tau)$, that turns out to be a left contact discontinuity with left state $u_{n, L}(t)$ and right state $u_{n, R}(t)=\left(u_{n, L}(t)\right)^{\sharp}$, we can define the lines

$$
\xi_{n, t}(\tau)=\varphi_{n}(t)+f^{\prime}\left(u_{n, R}(t)\right)(\tau-t),
$$

and then $w_{n}$ as

$$
w_{n}\left(\xi_{n, t}\left(t_{n}\right)\right)=u_{n, R}(t) .
$$

If the lines $\xi_{n, t}$ do intersect in $\left.] 0, T\right]$, then their tangent envelope is a convex curve $x=$ $\varphi_{n+1}(\tau)$ starting at time

$$
t_{n+1}=t_{n}-\frac{1}{\left.D_{x}^{-} f^{\prime}\left(w_{n}(x)\right)\right|_{x=\varphi_{n}\left(t_{n}\right)}} .
$$

Since in a solution $u$ to (1.1a) the maximal backward characteristic starting from a point $(T, \bar{x})$ with $u(T, \bar{x}+) \neq 0$ is a polygonal line with a finite number of nodes [10, Theorem 2.1], see Figure 7, we can hope to obtain a control $u_{c}$ in (1.1b) only if there exists $N \in \mathbb{N}$ such that

$$
t_{N}<0 \text { and } \varphi_{1}\left(t_{1}\right)+\sum_{i=1}^{N}\left(\varphi_{i+1}\left(t_{i+1}\right)-\varphi_{i}\left(t_{i}\right)\right)<b .
$$

Using the expression for $t_{i}$, such conditions can be rewritten as

$$
\frac{1}{D_{x} f^{\prime}(v(\bar{x}))}+\sum_{n=1}^{N} \frac{1}{\left.D_{x}^{-} f^{\prime}\left(w_{n}(x)\right)\right|_{x=\varphi_{n}\left(t_{n}\right)}}>T
$$

and

$$
\bar{x}+\frac{f^{\prime}(v(\bar{x}-))}{D_{x}^{-} f^{\prime}(v(\bar{x}))}+\sum_{n=1}^{N} \frac{f^{\prime}\left(w_{n}\left(\varphi_{n}\left(t_{n}\right)-\right)\right)}{\left.D_{x}^{-} f^{\prime}\left(w_{n}(x)\right)\right|_{x=\varphi_{n}\left(t_{n}\right)}}<b .
$$

Notice that the recursive procedure above does not allow for a complete treatment of the problem. This is related to the fact that, even at the first iteration of the procedure, we are not able to define the fictious profile $w_{1}$ on the right of $\varphi_{1}\left(t_{1}\right)$, see the proof Lemma 3.3 for an example in this direction. More in general the recursive procedure appears to be related to the a non-standard boundary value problem.

Let $N$ the minimum positive integer such that (5.10) (or, similarly, (5.11)-(5.12)) holds. Call $x=\gamma(\tau)$ the polygonal line defined by (see figure 7 , where $N=4$ )

$$
\gamma(\tau)= \begin{cases}\eta_{\bar{x}}(\tau) & \text { if } \quad t_{1}<\tau \leq T, \\ \xi_{n, t_{n}}(\tau) & \text { if } \quad t_{n+1}<\tau \leq t_{n}, n \leq N-2, \\ \xi_{N-1, t_{N-1}}(\tau) & \text { if } 0 \leq \tau \leq t_{N-1} .\end{cases}
$$




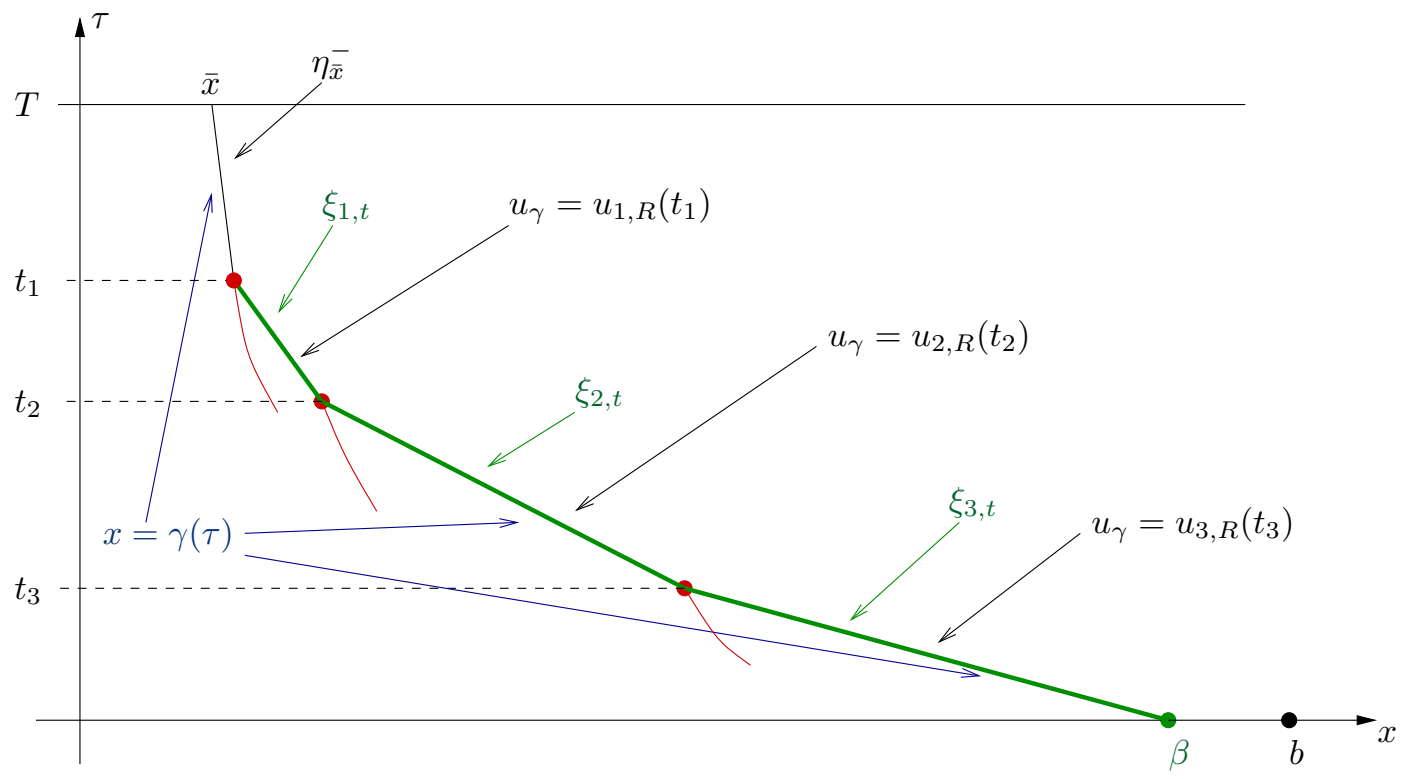

Figure 7: An open problem

Let $\beta=\gamma(0)$, so that condition (5.10) ensures that $\beta<b$. Observe that, once such a backward construction is done, the values that a candidate solution $u$ assume along $\gamma$ are fixed. Let

$$
u_{\gamma}(t)= \begin{cases}v(\bar{x}-) & \text { if } t_{1}<\tau \leq T \\ u_{n, R}\left(t_{n}\right) & \text { if } t_{n+1}<\tau \leq t_{n}, n \leq N-2, \\ u_{N-1, R}\left(t_{N-1}\right) & \text { if } 0 \leq \tau \leq t_{N-1} .\end{cases}
$$

We must find conditions on $v, \gamma$ and $u_{\gamma}$ such that there exists a control function $u_{c}:[\beta, b] \rightarrow \mathbb{R}$ such that the solution $u=u(t, x)$ to

$$
\left\{\begin{array}{l}
\partial_{t} u+\partial_{x} f(u)=0 \\
u(0, x)=\left\{\begin{array}{lll}
u_{c}(x) & \text { if } & x \in[\beta, b] \\
0 & \text { if } & x>b
\end{array}\right. \\
\lim _{x \rightarrow \gamma(t)^{+}} u(t, x)=u_{\gamma}(t),
\end{array} \quad t \in[0, T], x>\gamma(t),\right.
$$

fulfills $u(T, x)=v(x)$ for any $x>\bar{x}$. Observe that (5.13) is not a standard hyperbolic boundary value problem, since we do not prescribe simply a Dirichlet datum along the characteristic boundary $x=\gamma(\tau)$, but we impose the value of the trace of the solution. It is straightforward that some kind of compatibility conditions on $v, \gamma$ and $u_{\gamma}$ must hold. Indeed, at each node $\left(t_{n} \gamma\left(t_{n}\right)\right)$ of the polygonal line a left contact discontinuity must arise, and hence $v$ has to suffer from jump discontinuities in the half line $] \bar{x},+\infty[$. Moreover, in the limit case $\beta=b$, there can only be only one final profile $v$ defined on $] \bar{x},+\infty\left[\right.$ compatible with $u_{\gamma}$, since in such a 
case the solution $u$ to (5.13) turns out to be unique, and can be computed, e.g., by means of a front-tracking algorithm (e.g., see [11, Section 14.1]).

\subsection{A perspective research direction}

The solvability of problem (5.13) in the general setting is a challenge well beyond the scope of this paper. However, starting from the recursive procedure introduced above, it should be possible to obtain a numerical algorithm testing attainability of profiles associated to simple cases of nesting.

For any $T>0$, we consider the set $\mathcal{A}_{T}$ of all profiles verifying the assumptions of Theorem 2 for attainability at $t=T$. Obviously, if $T_{1}<T_{2}$, then $\mathcal{A}_{T_{2}} \subset \mathcal{A}_{T_{1}}$. Assume that $v$ is an attainable profile associated to a nested structure (see figure 6). We start the backward reconstruction of the profile $v$, as described in Section 3 up to the fist time at which nesting occurs, $\left.\tau_{1} \in\right] 0, T\left[\right.$ ( $t_{2}$ in figure 6 ). Of course, there holds $v \in \mathcal{A}_{T-\tau_{1}}$. Assume now that the nesting structure detected at time $\tau_{1}$ occurs on the right of a left contact discontinuity traveling along the curve $t \mapsto \varphi_{1}(t)$ (see figure 6) and that (this is a crucial hypothesis)

(NH) no nesting occurs in the portion of plane $\left\{(t, x): t \in[0, T], x \leq \varphi_{1}(t)\right\}$.

Then we can use the idea of the recursive procedure to construct a fictitious profile $v_{1}$ at time $t=T$. The profile $v_{1}$ is defined piecewisely as

1. $v_{1}\left(\xi_{1, t(x)}(T)\right)=v^{\sharp}(x)$, for $x \leq \xi_{1, t_{1}}(T)$;

2. $v_{1}(x)=v^{\sharp}(\bar{x})$ for $\left.\left.x \in\right] \xi_{1, t_{1}}(T), \bar{x}\right]$.

3. $v_{1}(x)=v(x)$ for all $x>\bar{x}$;

In such a way, if we apply the procedure of the backward reconstruction of Section 3 starting from the profile $v_{1}$, we obtain a solution $u$ which does not contain the left contact discontinuity $x=\varphi_{1}(t)$, and no nesting occurs at the left of $x=\varphi_{2}(t)$. Such a solution $u$ is defined up to $\tau_{2}<\tau_{1}$, where a nesting configuration may well appear again. In any case, $v_{1}$ belongs to $\mathcal{A}_{T-\tau_{2}}$. W We can iterate this procedure, and, if the analogous of the (NH) hypothesis holds at each step, we obtain a decreasing sequence of times $\tau_{k}$, and a sequence of profiles $v_{k}$ belonging to $\mathcal{A}_{T-\tau_{k}}$.

The explicit verification of the set of properties imposed by this construction (basically, one should check the assumptions of Theorem 2 at each step, plus a condition similar to (NH)) seems extremely expensive in practice. Nonetheless, these assumptions illustrate well the nature of the backward resolution of the non-convex conservation law (1.1a), where solutions should be constructed by piecing together patches of backward and forward solutions, as in the proof of Lemma 3.3. Numerical experiments with this version of the recursive procedure will be considered elsewhere.

\section{Acknowledgements}

The results contained in the paper have been obtained while the third author was visiting the Laboratoire de Mathématique of Besançon in spring 2015 as visiting professor. The third 
author is really grateful of the kind invitation and appreciated a lot the warm hospitality he received. Our results have been finalized during a one year visit of the second author to the Department of Mathematics of the University of Padua (projet Mobilité sortante des chercheurs 2015, Région Franche-Comté).

\section{References}

[1] Adimurthi, Shyam Sundar Ghoshal, and G. D. Veerappa Gowda. Optimal controllability for scalar conservation laws with convex flux. J. Hyperbolic Differ. Equ., 11(3):477-491, 2014.

[2] Adimurthi, Shyam Sundar Ghoshal, and G. D. Veerappa Gowda. Exact controllability of scalar conservation laws with strict convex flux. Math. Control Relat. Fields, 4(4):401-449, 2014.

[3] Fabio Ancona and Andrea Marson. On the attainable set for scalar nonlinear conservation laws with boundary control. SIAM J. Control Optim., 36(1):290-312 (electronic), 1998.

[4] Boris Andreianov, Carlotta Donadello, Shyam Sundar Ghoshal, and Ulrich Razafison. On the attainable set for a class of triangular systems of conservation laws. J. Evol. Equ., 15(3):503-532, 2015.

[5] C. Bardos, A. Y. le Roux, and J.-C. Nédélec. First order quasilinear equations with boundary conditions. Comm. Partial Differential Equations, 4(9):1017-1034, 1979.

[6] Raimund Bürger and Wolfgang L. Wendland. Sedimentation and suspension flows: Historical perspective and some recent developments. Journal of Engineering Mathematics, 41(2-3):101-116, 2001.

[7] Marianne Chapouly. Global controllability of nonviscous and viscous Burgers-type equations. SIAM J. Control Optim., 48(3):1567-1599, 2009.

[8] Corghi, Marco and Marson, Andrea. On the attainable set for scalar balance laws with distributed control. ESAIM: COCV, 22(1):236-266, 2016.

[9] Jean-Michel Coron. Global asymptotic stabilization for controllable systems without drift. Math. Control Signals Systems, 5(3):295-312, 1992.

[10] C. M. Dafermos. Regularity and large time behaviour of solutions of a conservation law without convexity. Proc. Roy. Soc. Edinburgh Sect. A, 99(3-4):201-239, 1985.

[11] Constantine M. Dafermos. Hyperbolic conservation laws in continuum physics, volume 325 of Grundlehren der Mathematischen Wissenschaften [Fundamental Principles of Mathematical Sciences]. Springer-Verlag, Berlin, third edition, 2010.

[12] O. Glass and S. Guerrero. On the uniform controllability of the Burgers equation. SIAM J. Control Optim., 46(4):1211-1238, 2007. 
[13] S. Guerrero and O. Yu. Imanuvilov. Remarks on global controllability for the Burgers equation with two control forces. Ann. Inst. H. Poincaré Anal. Non Linéaire, 24(6):897906, 2007.

[14] David Hoff. The sharp form of Oleĭnik's entropy condition in several space variables. Trans. Amer. Math. Soc., 276(2):707-714, 1983.

[15] T. Horsin. On the controllability of the Burgers equation. ESAIM Control Optim. Calc. Var., 3:83-95 (electronic), 1998.

[16] Helge Kristian Jenssen and Carlo Sinestrari. On the spreading of characteristics for nonconvex conservation laws. Proc. Roy. Soc. Edinburgh Sect. A, 131(4):909-925, 2001.

[17] G. J. Kynch. A theory of sedimentation. Trans. Faraday Soc., 48:166-176, 1952.

[18] Matthieu Léautaud. Uniform controllability of scalar conservation laws in the vanishing viscosity limit. SIAM J. Control and Optimization, 50(3):1661-1699, 2012.

[19] Vincent Perrollaz. Exact controllability of scalar conservation laws with an additional control in the context of entropy solutions. SIAM J. Control Optim., 50(4):2025-2045, 2012 . 